\title{
ชก2h4 กU4Uน3u乙
}

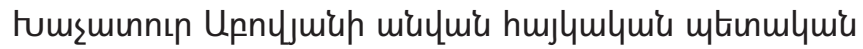

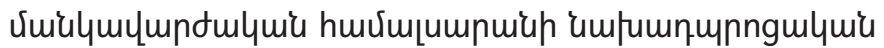

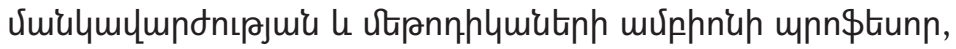

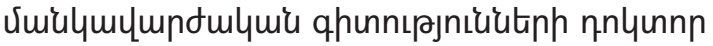

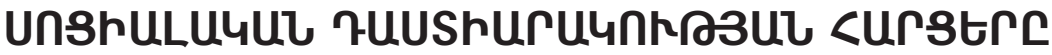

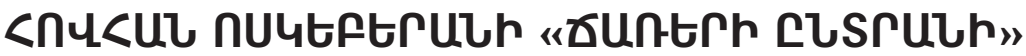

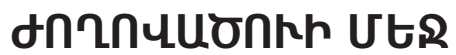

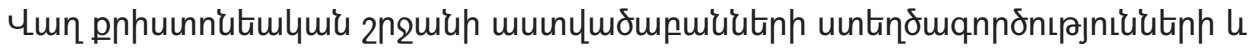

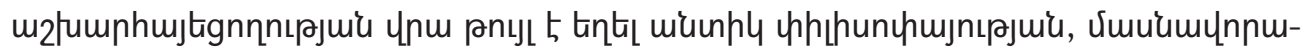

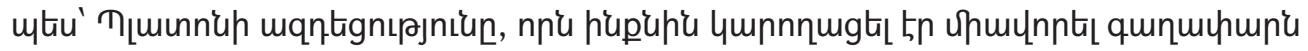

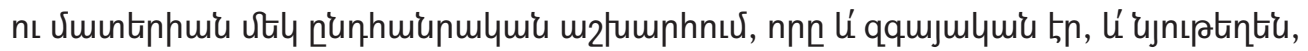

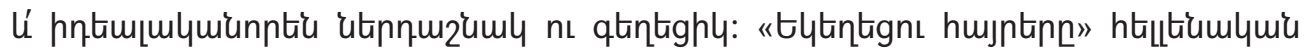

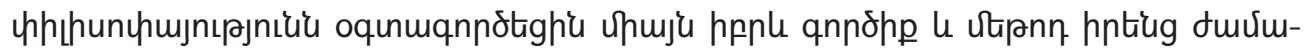

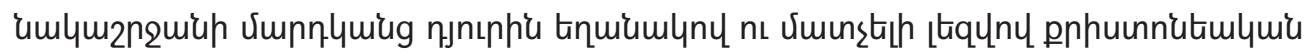

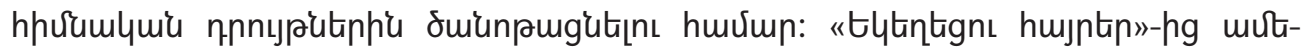

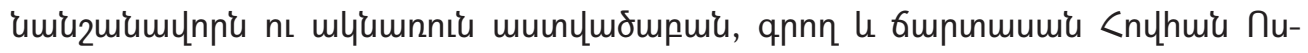

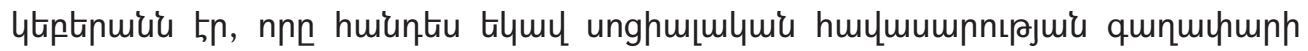

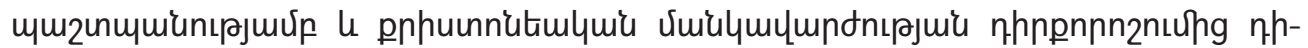

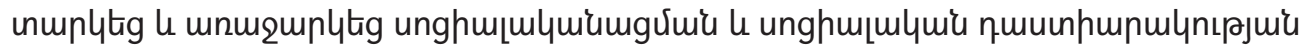
hungtip:

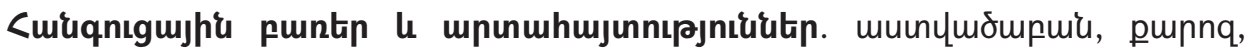

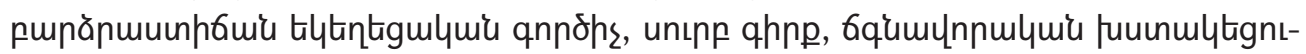

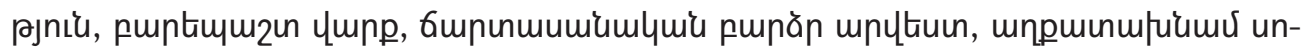

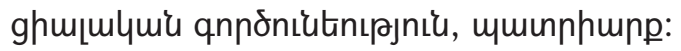

Fjntquiunulymì punănumunh6uís tyltintigulyuiu qnodps, qnnn u hntiunnn

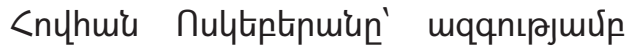

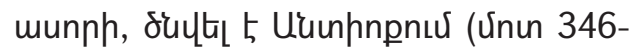
407 рә.)' hupniuin pnpuunnitumluie

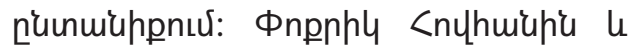
unu dunn'utphe' hpple pphuinn'uju vilnunt 5 4nuinuiun'unsunjuh umunnhump

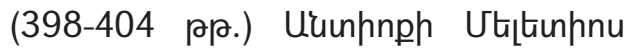
hujnumbinn' mqqnıpjuर्up hus: Unm hnцाumunnnıрjuर्up <nuhuiun unun-

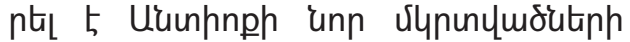

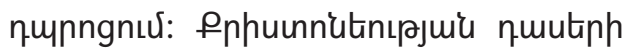
htien vityontin duviuimuly azmiumunn pưuuunuutpitiph le hntiunnnutiph ưnun

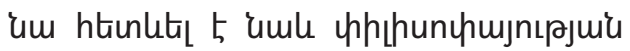

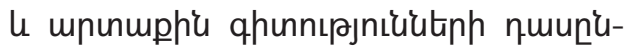
pugutphis, ununnti unuhtiuun Lhpuiun-

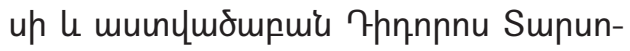
umgnı unun: Ujunshtiunle Uptiupnıu uhp-

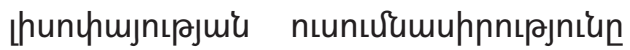

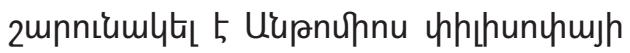




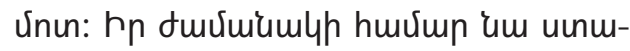

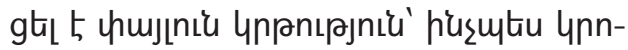
umlquiu, mjumbu th m2tumphnly: Utio quiumunnnıрjuर्up nı htinlunnulquiun-

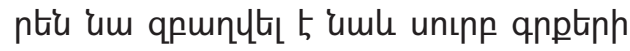

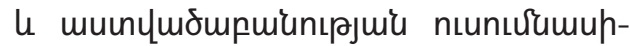

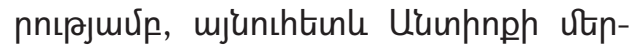

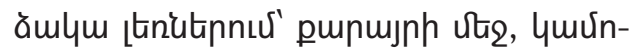

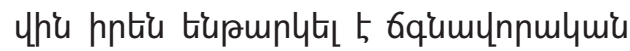

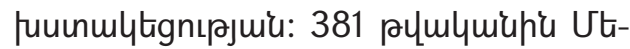
jtinnnu humpumutionh ynnưhg üu ătin-

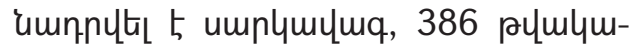
un'i puhwium, nnhg htinn lumpltat 5 Uuunnnph qutumunn tutantigns punnqhe: «ty n'y yunnn 5 unu zunnhutiph

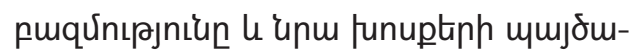

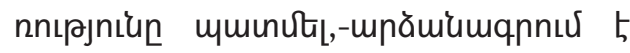

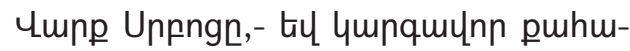

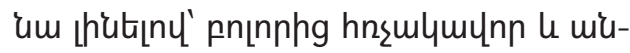

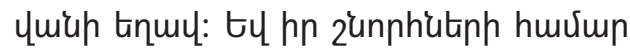
tuphulnumnunıpuiu muinlh ynsutig

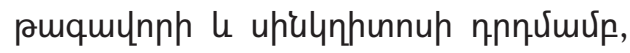

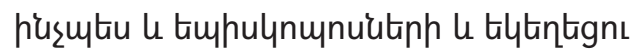

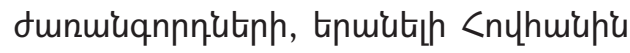
40 unmptiquiunux 4. Tnjup timpulnmenu

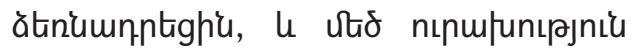

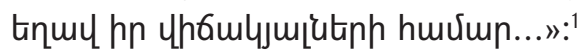

funtimuzun ympph lu unuphiunt-

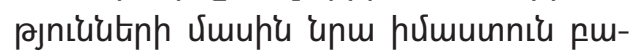
umfununıpnıüutipis nı unıp qppt-

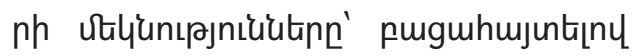

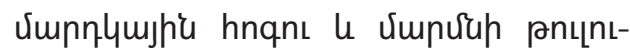

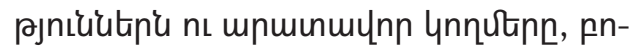

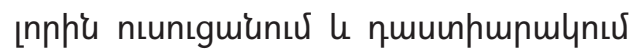

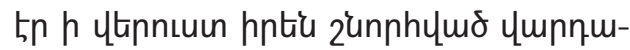

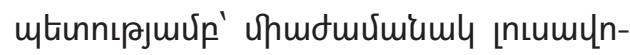

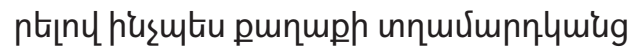

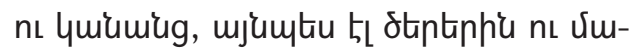

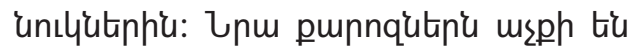

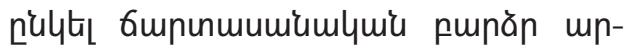

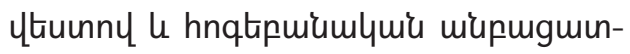

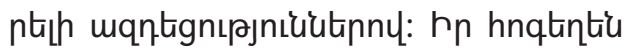

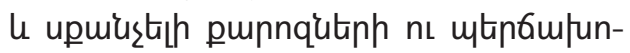

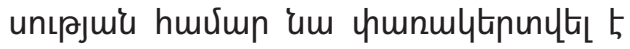
"nulyh ptrpuiu» muinuluiunı'uny:

<nyhuiu nulteptinuiun hnulymiamg-

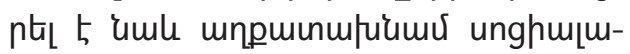

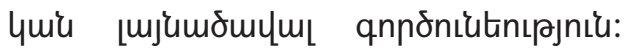
Uuunnnph tyltintgnnu unm mưhqu-

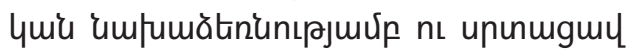

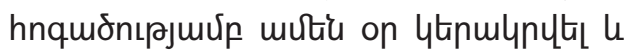

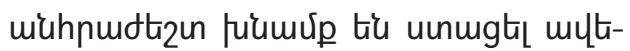

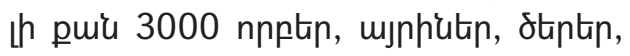

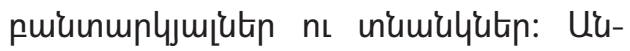

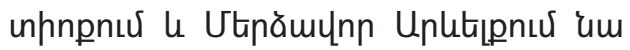

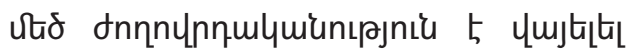
humnsuunutiph gnih nเ 24ujun ytiugu-

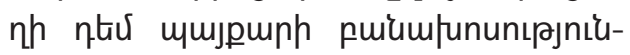

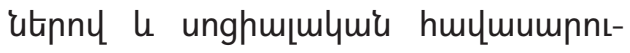

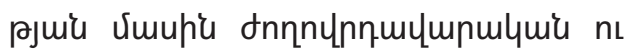
vimnmunnmumiu pupnquitpny: $\rho_{u-}$

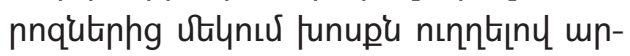
pniuhph'u, punănuuunh6uiu tilting-

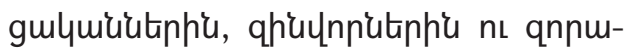

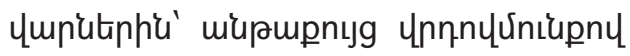

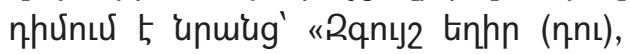
nn mנn funphnnh unten tu. puquelnn

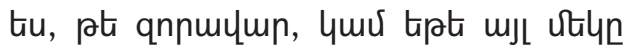

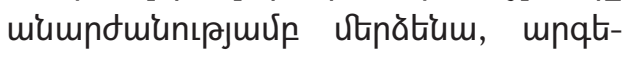

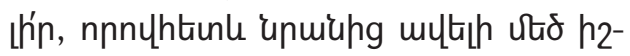

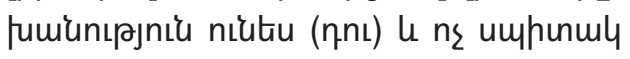

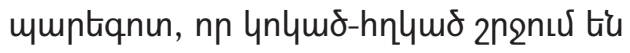

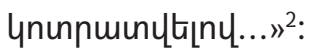

398 pumlumupre <nuhwir nultektnuiun hnuulhnytas 5 4numuiun'unımnhu

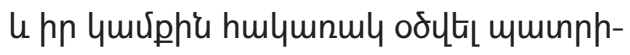

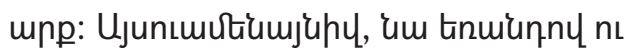

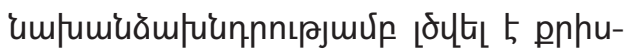

1. Umpp Unnng, huinnn ৭, U. tquhmirhü-2010:

2. Unแยน untinnเư: 
unnutumlymí ltiugunng htnuguro wiu-

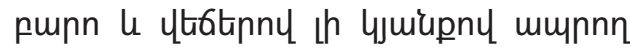

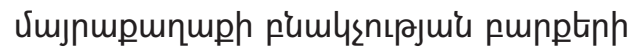

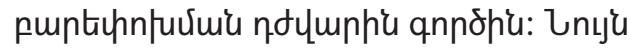

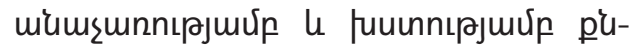

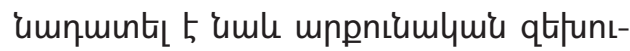
pjniưutinn: Ruiumgtal 5 uuntindta 4nu-

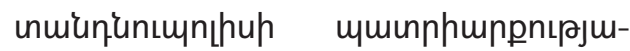
un tiupmlym unptiqtinulymirs tiltintigh: Uphuinnlynuinhujpha, mpnniuhphe $u$

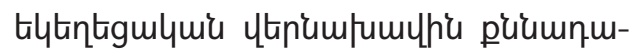

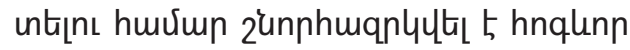
ynsnuing, hul 403 pululuming funlyt-

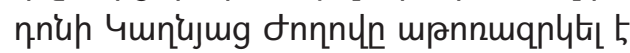

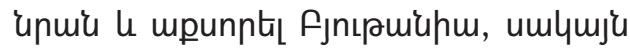
utily on ming onnnunnth huulum muhwiugny um unnng ytanununăta 5 4nu-

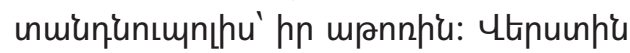

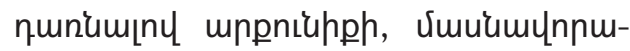

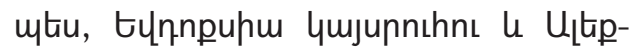

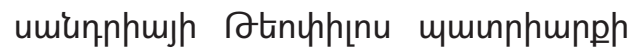
jumpnulu'uputiph qnh' 404 pumlquuna' hulqunmul onnnunnh ymúppha $\mathrm{l}$ Upluvinjuir tiltintignı hujptiph mm2unumiunıpjuiun, Uplqun-pnu qujutin hnuxumiuny upunnlts 5 बnpn <ujph unlhun's punupg, nnuntin vaugtal 5

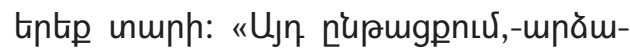

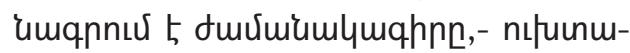

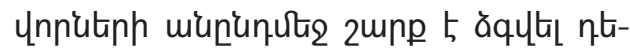
un unlhunup hus tlytintigh: Struitinny

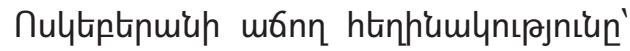

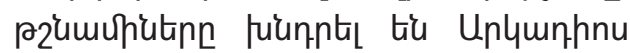

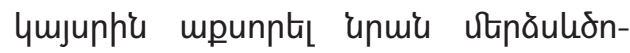

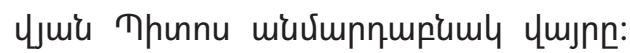

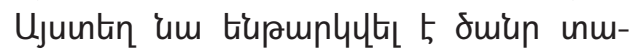
duiumlynnıpjuiu. gnınun ăưnuiun Guium-

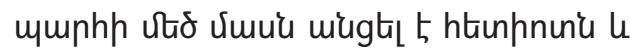
Tniunnup 4nưuium punuph unin, up-

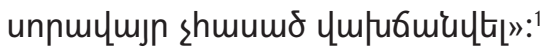

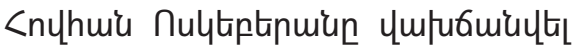

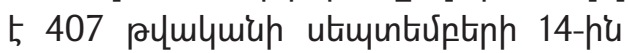

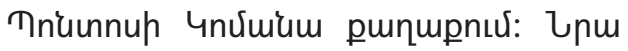
vimhng 35 unwph ming 4nuinuiununtunnup unnnuunpr muinnhwnp Tpnl-

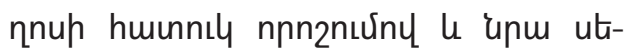

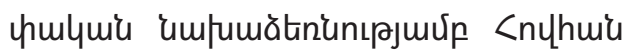

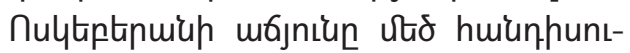

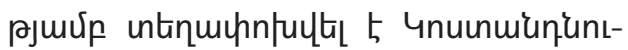

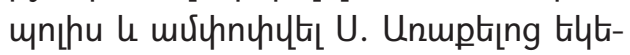
$\eta$ tigntu: ${ }^{2}$

<nyhuiu nulytptipuiun pnnts 5 umintiumqnulymir hupniuin dunuiugnt-

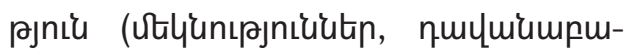

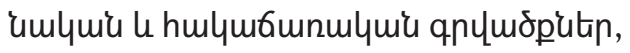

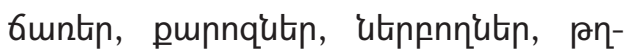

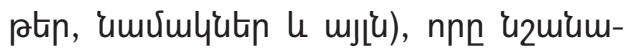

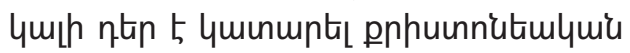

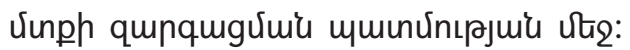
unu trytinu nı qnnotinu hntiug ălumpn-

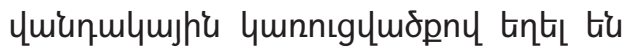
ungiumpunjuis hntionnnulymir funuph $\mathrm{l}$ n6h, umb tlytinggulymín ptrúpmumgnt-

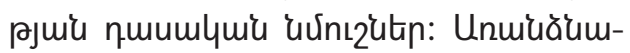
mbiu nizmqnuy tíu unm pupnjm|unumyuir Gunntinu nt hnnnnnulyitinn (hteqnt-

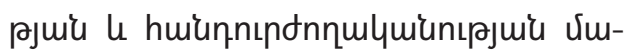

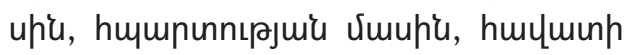
vimupu, uppn vimupu, unnpph vimupu,

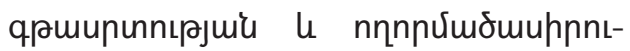

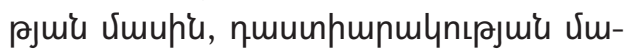

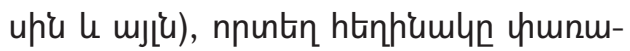

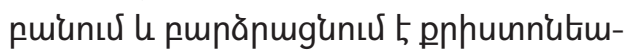

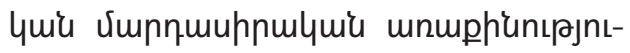

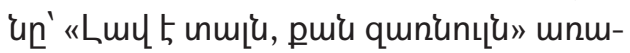
ptimlquiu ulqqpniupny: 7pu huvium ium

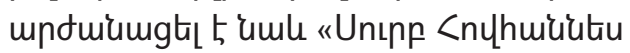

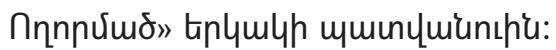

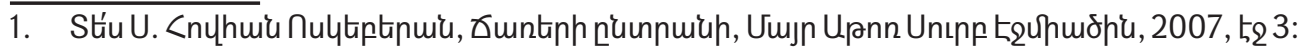

2. Stúu «fphuunnuju <mjuuunuiu» hưunuqhunmpuiu: 


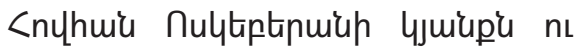
vimutiumqnulymir ymunnuly puq-

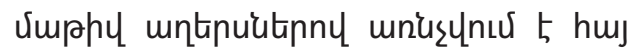

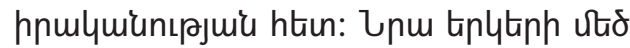

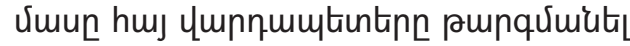

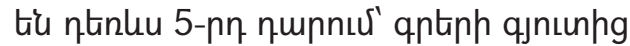

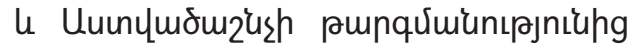
miuungmutiu htionn: Drunquiuiunıpjniu-

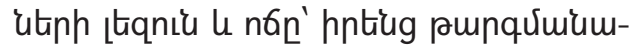

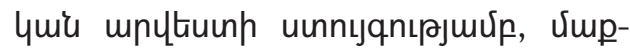
nnıрјuर्up nı qtintiglnıрјuर्up, hnu-

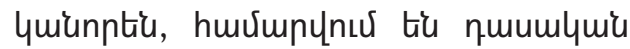
punquimiunıpjnıüutip:

fiumplitiny unghm[-ปmilymump-

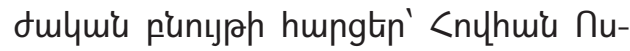
ytaptapuiun ălumpnymianmulymphu unnt-

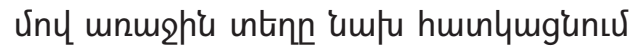

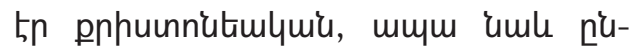

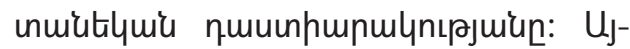

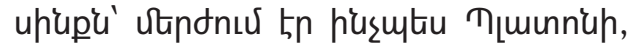

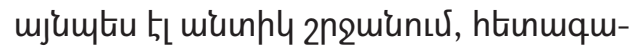

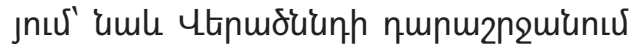

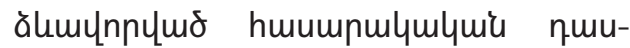

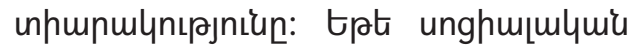

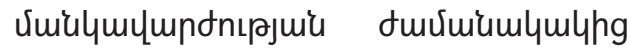

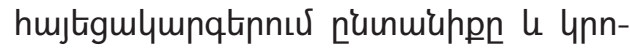

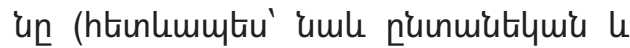

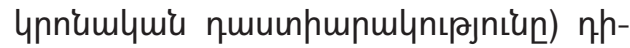
unuplyunư tiu hpple unu'uăhư unghujulquí qunnnghitin, mum nultiptipu-

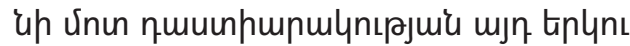
punqunnuiumutinn (htinlummtiu' 'umb

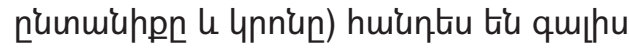
upmup's' hpple utly upmu'amlymir mu-

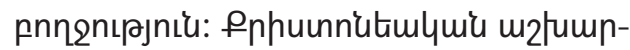

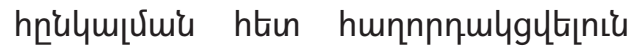
tinthumjhu utiunp 5 oqutiu dunnutitn, tytintigulquiukitinn u huviujupn: Ujuonhumuly ung'unnnuuपnnumo m2łumphn'uymuluiu ungngny tiptipumiting mtinp

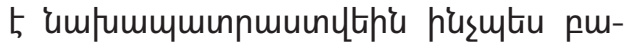

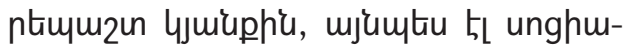

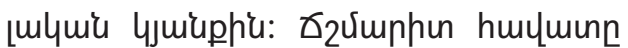

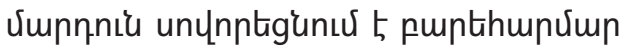

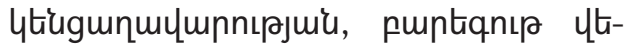

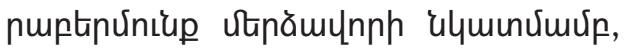

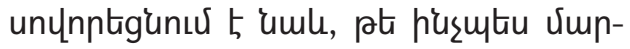

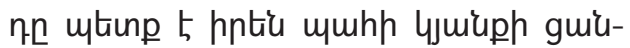

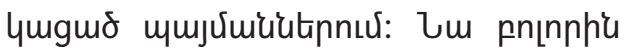

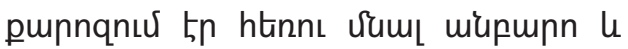
up'u hu6nıpgutiphg, puiuh np «Uuunonı'u

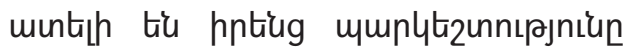
lnngunnutitnn....:

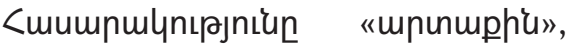

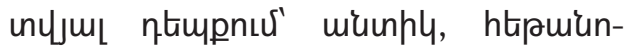
umlquir a pnhuunnutimlymis quuunhw-

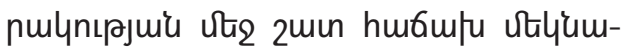

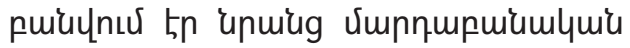

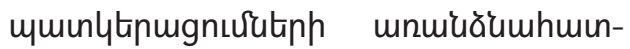

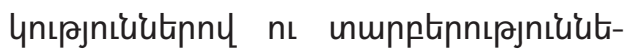

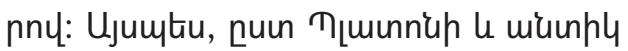

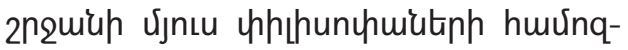
uniuputitnnu, tipt $\mathrm{h}$ ulqpuits vimnnn

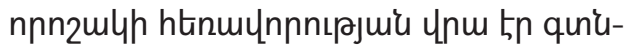
Unıu muunumoutitphg, nıptiư hn ungh-

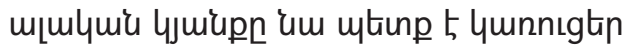
utihulymí nıdtinny, hn unmugur mp-

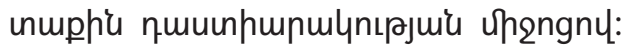
rusustu <nyhuiu nultiptipuiuh, mju-

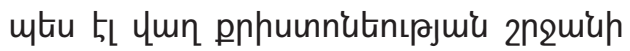

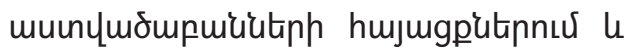

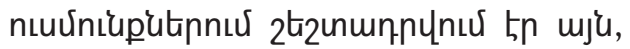
np huumpulymlymis unmpptip husunm-

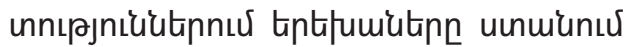
the unuly m2łumphply unpnıpjniu, upurz-

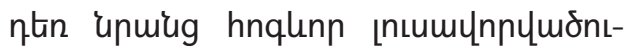

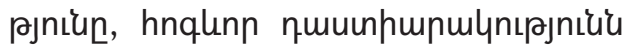

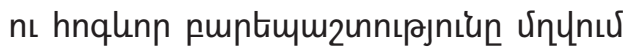
tn htinhis mimis: Ujumpuny' ptimtin ymn pnhuunnitimlymi vimlymumpont-

1. Stíu Uminp Unnng, humnn ৭, U. Łquhurghiu-2010: 


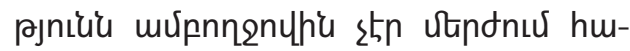

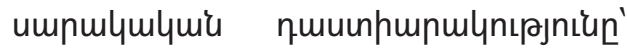
unu utig untüutisny 'umle nughn'um! hu-

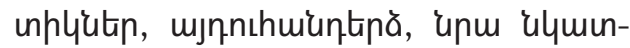

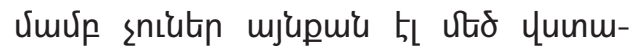

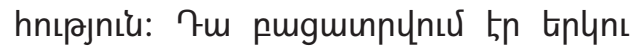
munn muinfiunny. 'umpu' 2 min hnqunn

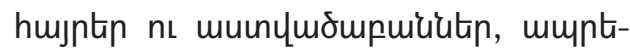

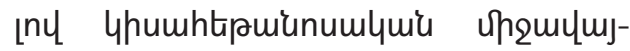
nnuर, hnurumghnntiu tiupounpnus thu,

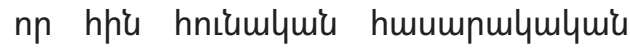

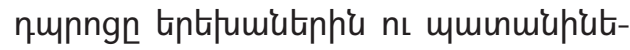

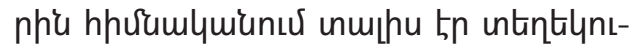

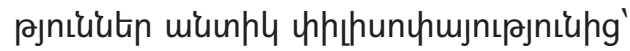

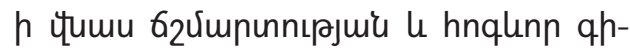

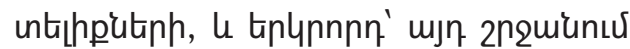

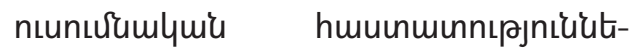

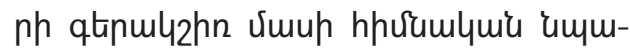
unmly humumulynıpjuíu utis huunicu

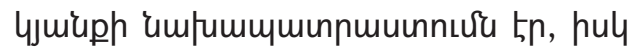

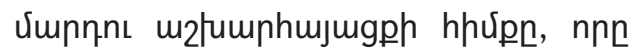
pnhuinnutumluiu untumialyniuny unm-

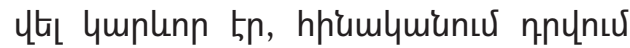

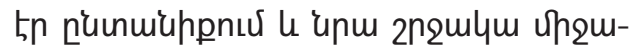
umjnnıu: Ujn muinfiunny th pphuinnuju dunnutitph $u$ hnqunn nuunıghšutiph

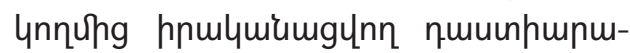

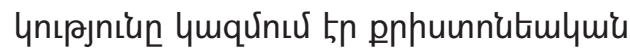

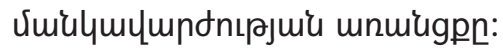

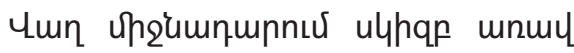

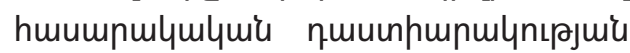
'u2míum

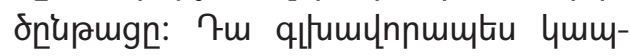
y wo th utinnıpjuir utis pnhuunnut-

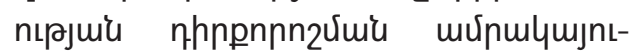

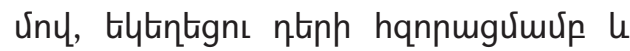

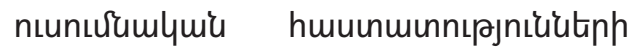

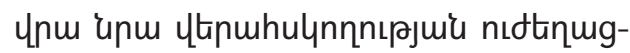

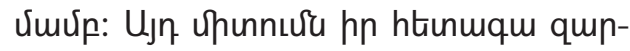

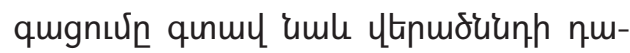

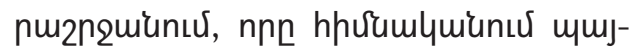

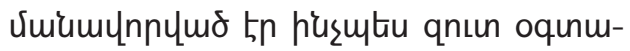

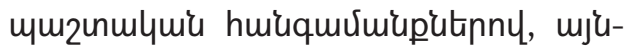
utiu th ununtiunıpjuí qunqugnnưny

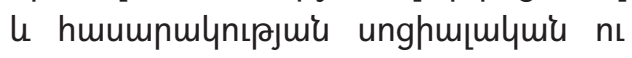

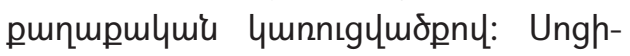

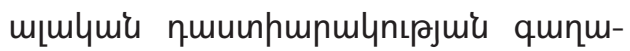

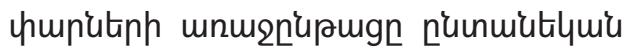

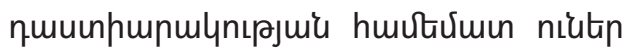

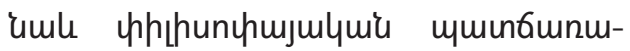

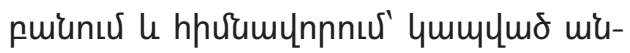

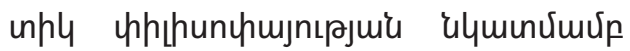

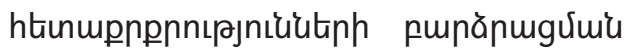
htin, unmeh'u htpph'u Tluunnuh, un-

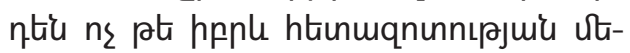

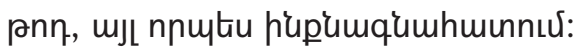

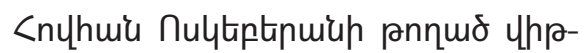
jumph dunuiugnıpnntúu hn unupnnnt-

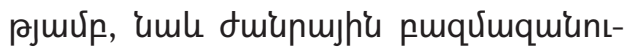

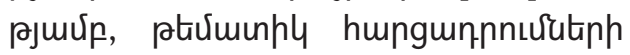

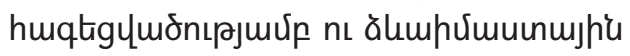

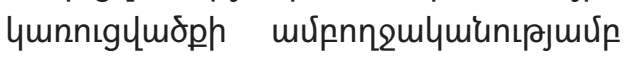
nı huर्umlympqumonıрjuर्up, hpnuulh, qtinuquiugnư 5 mju mutiuhu, h'us ưumgta tn ywn pnhuunnutimlumi 2nquiuh

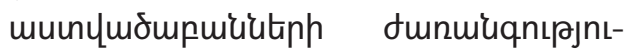
ung: uju mutiun, hius qpis no uuntinguqnnotal 5 'um lumpteh 5 qunutas l' unnüuquiunıpjníu, l' unn'umlymí qnulymiunt-

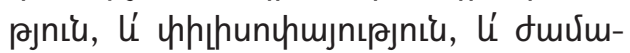

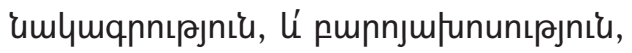

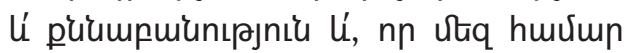
surhuquiug lumbinn 5, pnpuinnitim-

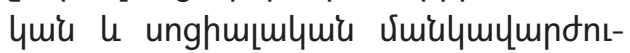

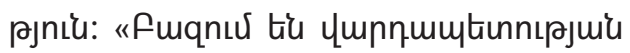

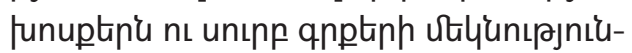
Utann,- qpnuर्u 5 Uump Unpngn,- nn tinuutip untip <nuhwiun' 4. Tnjup tumhuln-

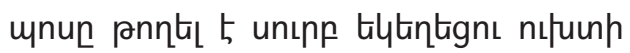

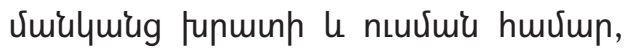

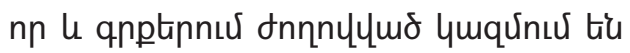
unmuktinlyns huqum Guntip, 'umle nnnugg 


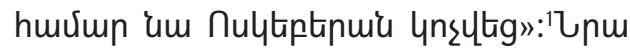

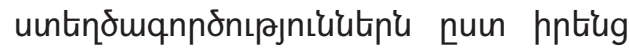

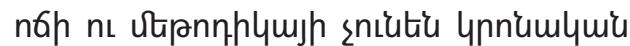

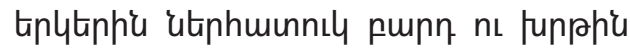
nGupuiunıpjniu, funupp $u$ vinph wiu-

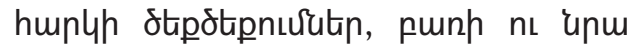

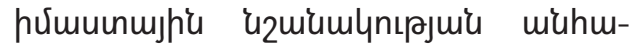

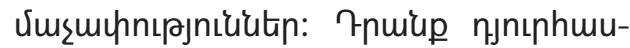

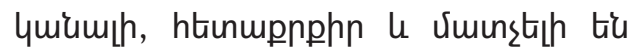

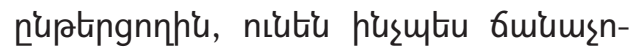

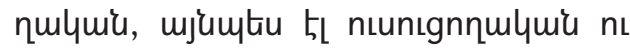

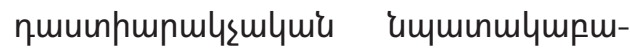
unเคjnเu:

<nųhuiu nulytptrpuiuh uuntindmqnn-

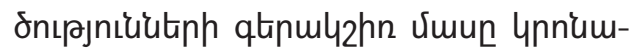
ywikng quin, unhmumpuly, ythnupt-

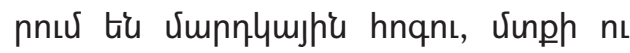

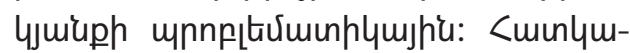
utiu nt2mqnuy tiu $u$ utio htinmpnpnnt-

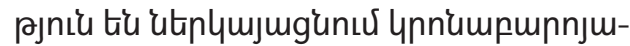
junumlymí pnцmïnulynıрјuй unm 6unting, nnuntin un ynnưhg um puiumpu-

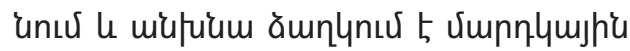
mjumpup unptin, mpuinutin nı un-

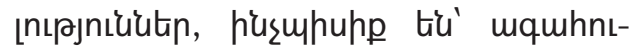

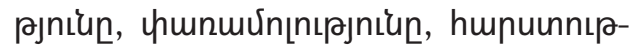

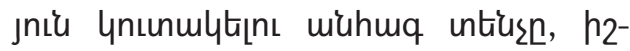
fumiumuntiušnıpjniun, umfumiuăn, quqqnufununıpjnı'u'u nı puiuumplynıpjniun,

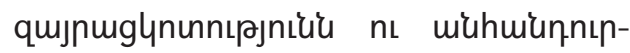
¿nnulymiunıpjniun, hul vjnıu lennuhg,

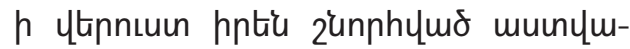

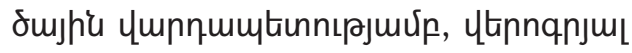

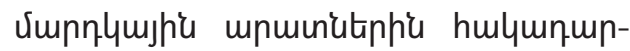

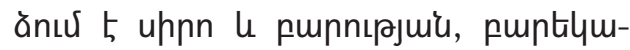

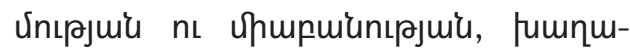

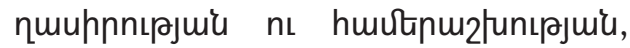

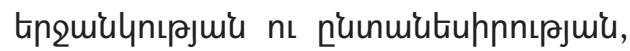

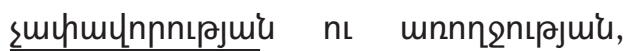

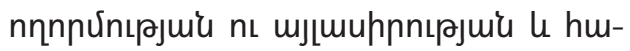
vuviumnlqujh'u mJ punănundtip qu-

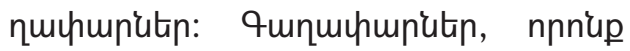

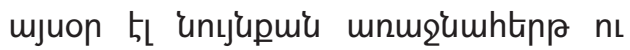

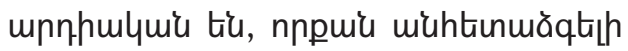

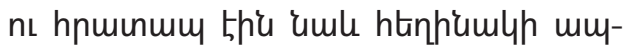

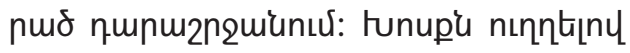
hn muinumup n'uptingnnhi' nuptiph ht-

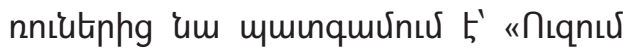

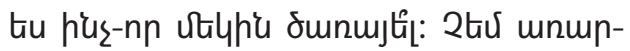

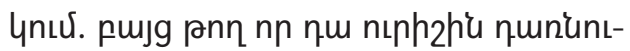

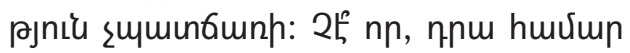

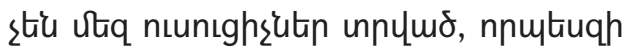
p2umưuiup niutiumiup upujuing huilu-

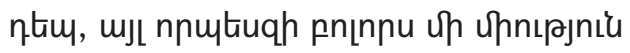

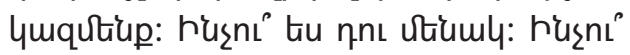

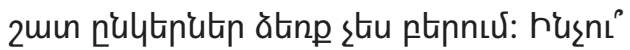

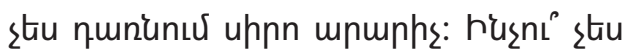

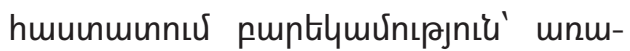

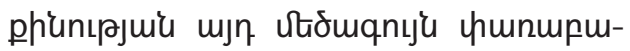
unเu็n:?

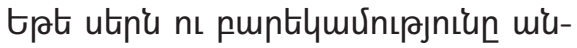

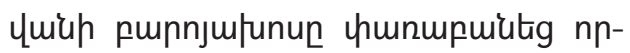

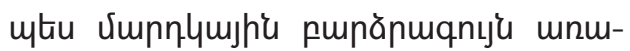

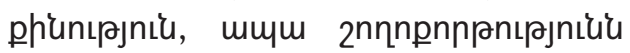
nı quqnumununıpjnín 'um hurưuntig

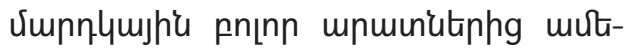

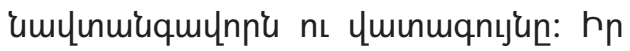

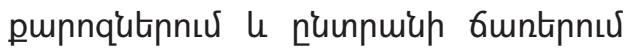

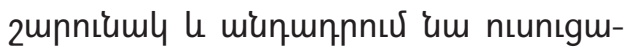

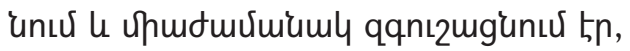

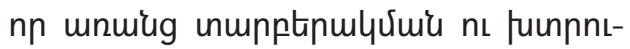

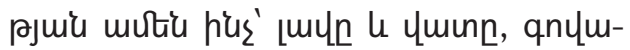

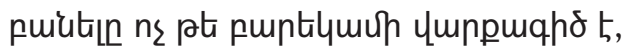
mJL' 2nпnpnnph nı quqnumunup, nüuhulqunmun, juц qnnos nnцmintįn $u$ ymun mpmpph munumulting um 5 62 nhen nulytinne lu puntiluuip mununpn.

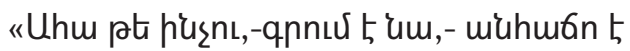

1 Umpp Unnng, huinnn 9, U. Equhurohu-2010:

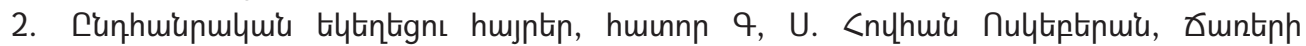
nuinnmuih, hnuin. U. Equhurohu-2007, t, 17: 


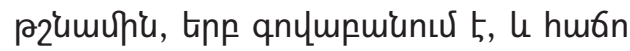

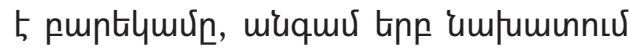
5 huă: しm, ny ptumtinl huúpnınnuर 5 puă, muhuran t5, um, ny ptrutionle yh-

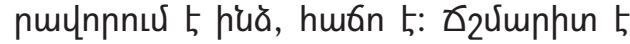

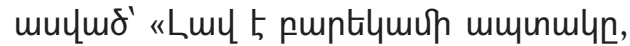

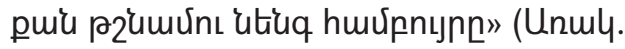
27, 6)»:1 Punnqtinny utip l puntilumưne-

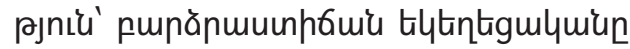
pme huưnqumo $5 n$, nn uppn nıdn huymumpuqnn 5 punısjuiu nıdhu, untunuly mutthé, nnnyhtinle mju, h'us nn sh

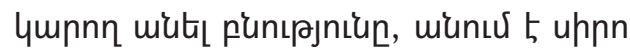

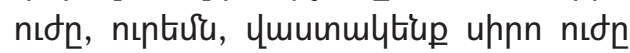
u npuiuny mumbnyltiup ulap hnqunn

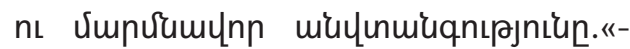

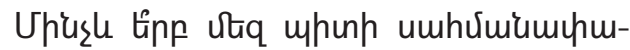
ytup utilyny ymu tinyniuny: Um ny ntup puquiuphy puptlymưatin, ptilnsqu unpwiun Lhuh, humnsuunutiphg mutth hupniuun 5 , has sh huiunque muts $h n$

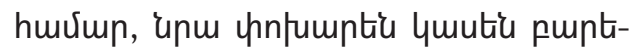
ymưutinn, hás sh yunnn ătinp ptintı hn huvump, ătinp uptiph vjncuiutiph zinnhम4»:2

husutiu T|nunumpnun, Multiptinu-

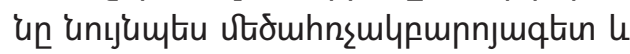

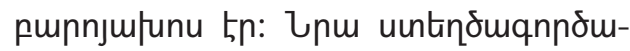

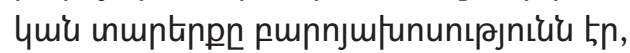

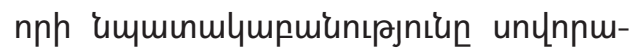

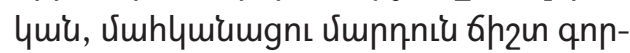

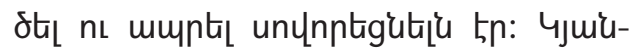
phis 'imfummminnmuinultint, mjuphupe'

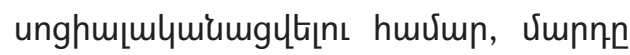

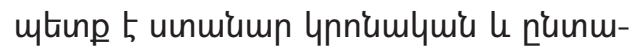

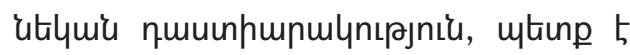

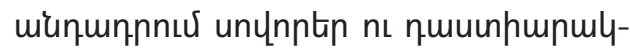

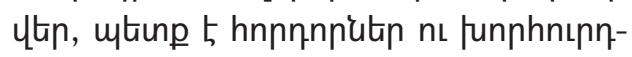

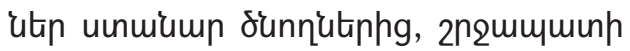

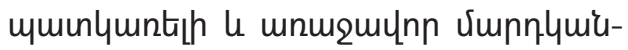

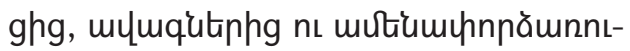

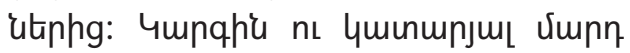

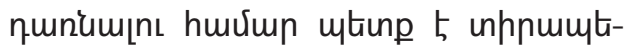

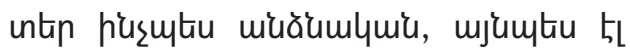

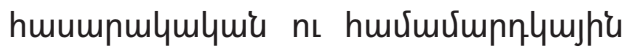
puquiuintumly punnjulquis undtip-

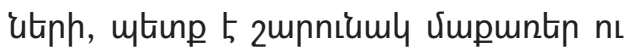

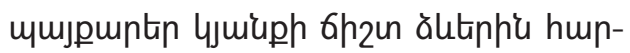

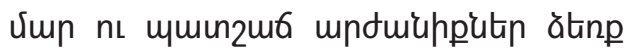
ptipt_nı huvium:

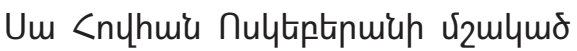
6h2un munt_nı 4Juiuph puiumăliu $5 n$ :

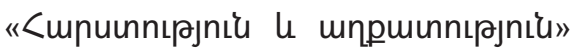

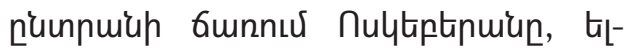

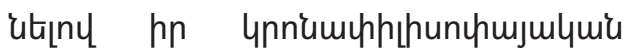

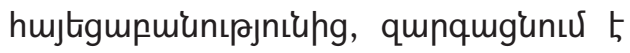

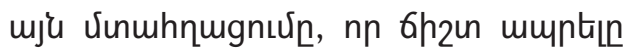

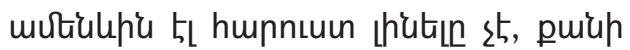

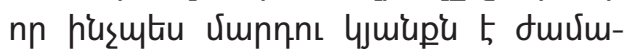
umlymunn ne tiupmlym vimhluiu, 6h2

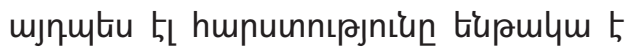

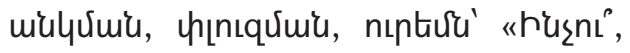
ńl Umpn, humuinnıpjniun ptiq guiu-

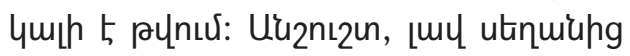
uunugur hu6nıjph huर्ump, múphnup u phliummuhitiph ynnung undwium-

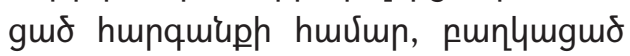

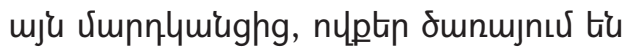

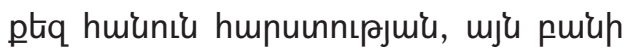

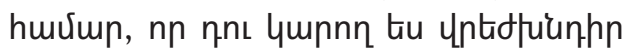
hiuts ptiq uhnulnnnnuting u ump-

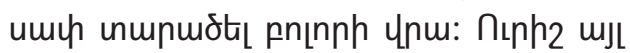

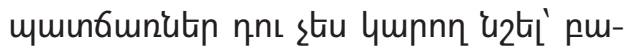
gh hw6nıpphg nı 2nnnpnnpnıpjniuhg, whhg nt Untuduünnnıßjniuhg»:3 Uunnt-

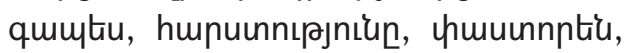

1. Unıju untinnư, to 19:

2. Unıju untinnư:

3. ᄂnuju untinnư, t2 84: 


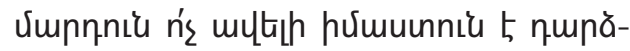
unus, n's mutth qnum, n's puphe, n's umpnuutin le unu hnqnı utig ns up mJt

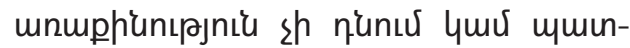

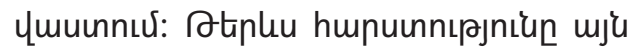
muinfiunny 5 "guiblymцh" ns "punăm-

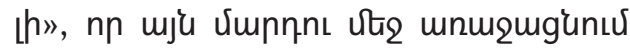

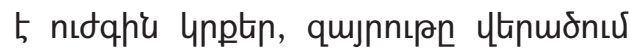
qnnoh, uhplumph sumhtiph huuguntu

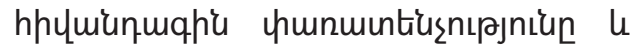

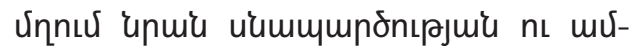

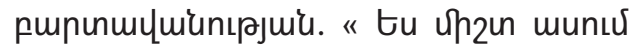

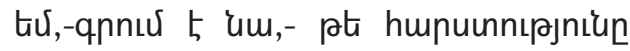

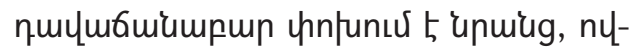
ptip yuin tiu oqunuqnnonnu mju. whm

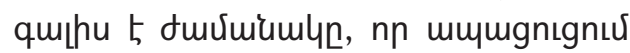
5 mjn funuptiph hnuulughnıpjniun:

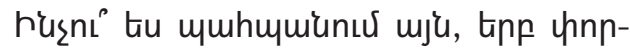

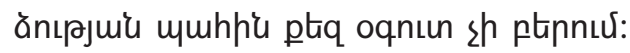
...huly tu humniuunitiphis hnqtihuiu tur

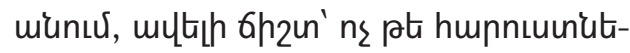
nhu, mJ unwing, nuptip stiu ymnnnu-

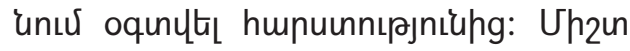

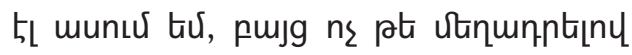
humniuunhiu, mJL qh2munshiu: UJl puir 5 hupnsuunn, mjı qh2munhen. ununptiphín mjn puitinn 4 un' fumnuhn miaunu-

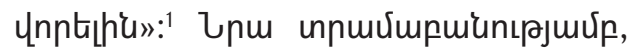

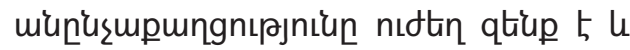

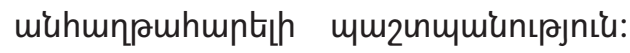
Ctinlumpun' unmju 'um, ny ulandta 5

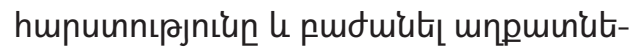

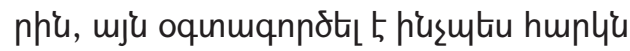
5 l htnmiaminy mju m2fumphing 'unu Unm nicutigts 5 quinumjul h2łumiuns-

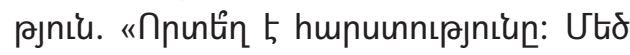

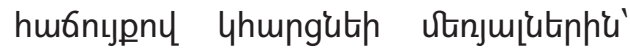
nnuntin 5 humuinnıpjniun: Um nnutiu

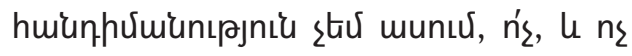

5L mju puiuh huviump, nn pnnpnptiu ythptinn, mJ mju muinfiunny, nnmtiuqh nıph2utiph unnomiuntưng ătiq huviump fumqun huiuqnumis muinnmuintus?: npulquiunus utiup pnınnu unuly oqunu-

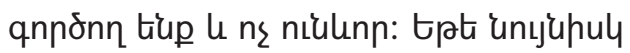
ulap nn2 4juiuph n'upugpnux hupuinnt-

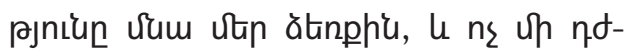

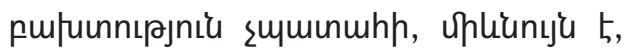

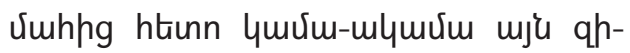

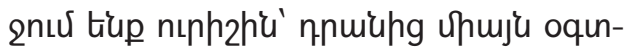

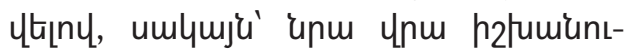

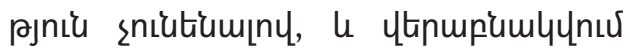

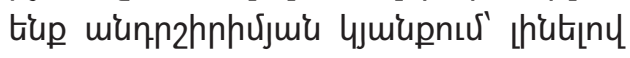

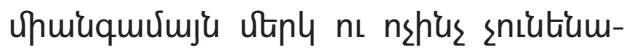

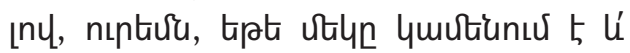

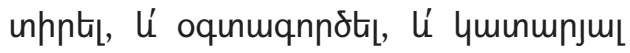

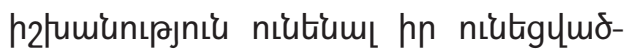
ph Unu, pnn tiptu ptiph guialymgur humuunnısjniuhg $u$ husphg, npnuhtiunl'

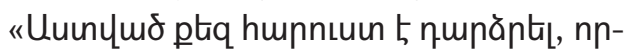

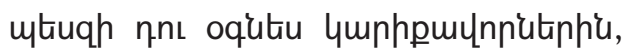

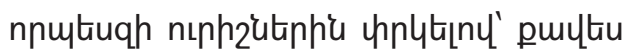
ulinptinn, ptiq unn 5 unltis ns mju puiuh

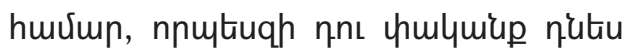

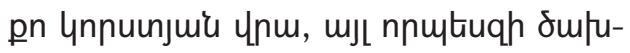

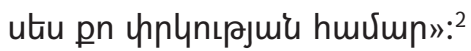

<nyhuiu nultiptipuiu' hn paumpu-

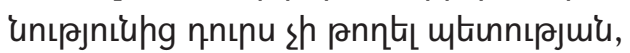

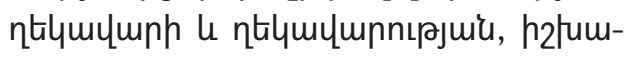

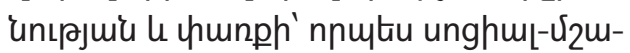

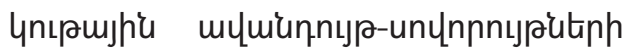

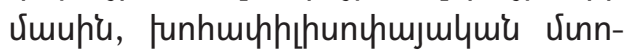

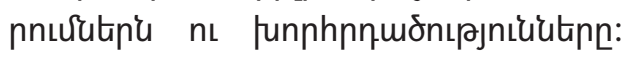
Dtt husuhupu mitung 5 hiutin puquuln-

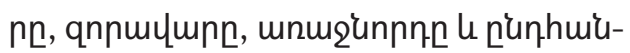

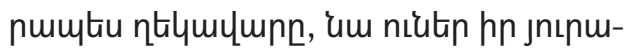

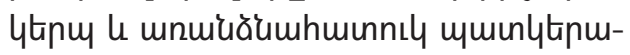

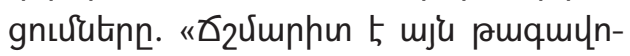

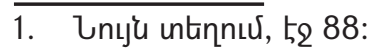

2. Unแju untinnux, t.2 100: 
nn,-qnnuर 5 'um,- nu h2łunuर 5 punlynt-

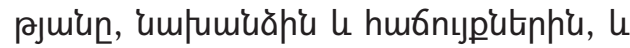
uuliú hius tüamplynư 5 Uunon optiup-

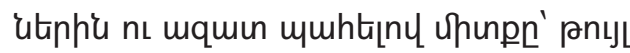
sh unmugu, np hnqnư pumlylh hu6nupph h2łumiunıpjniun, mјnuhup vimpnniu tiu

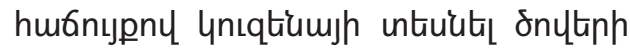
nı tnluph, punuputinh, onnnunınnitinh nı puiumlyutiph unmęnnn»:1

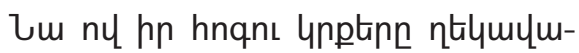

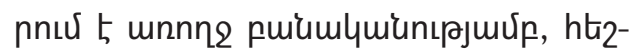

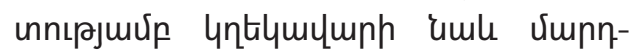

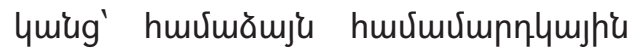
optiuputiph, mjumbiru nn, tiupmlquiukt-

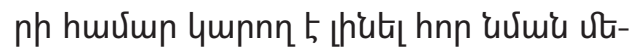

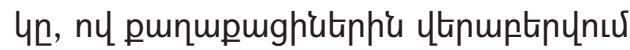

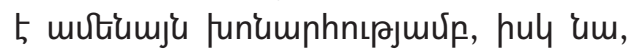
ny ununmpniuin h2tuntu 5 vimpnlyming \nu, pujg hiupn dunmunıu 5 qujnnı-

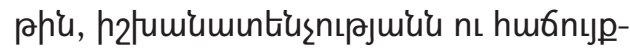

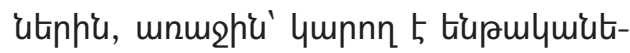

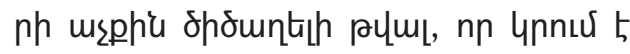
"puilquiqhe puntinny qunnunцmo

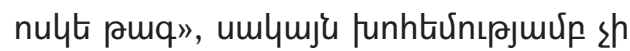

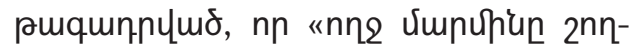

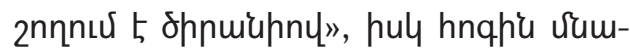

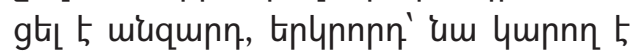
shưuium, pt huzustu unionhiuh h2lumunıpjniun, nnnyhtinl 'um, ny h yh6ulyh

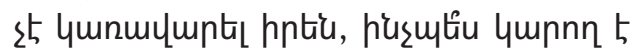
nıph2utinhu ymnwumptis ontiuputinny: Ttuing 5 ăquntă ns mju puiuhu, nn oqun-

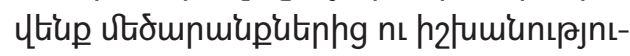
ung, uJ unuiu, nputiuqh thutiup unu-

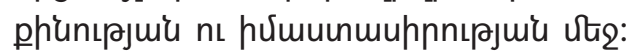

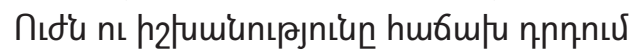

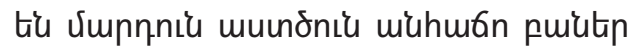

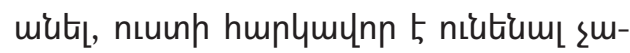
thuquing mph hnqh, npmliuqh ympn-

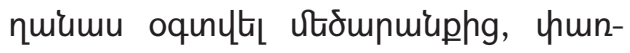
phg $u$ h2fumunıpjniuhg: $u m$ ny qnıpl

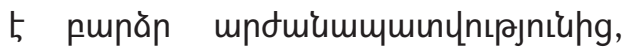
mlquर्um hơmunmulynu 5 , hul kim, ny

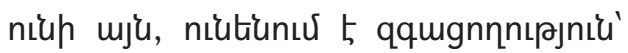

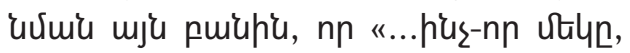
muntiny puptintiu le qtintighl ynujup

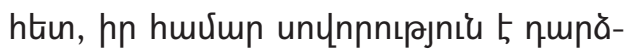

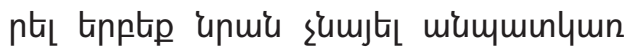

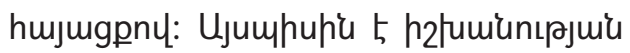
punıرคn»:?

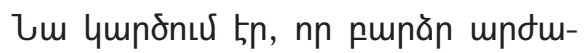

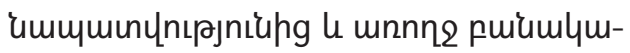

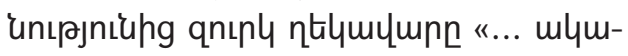

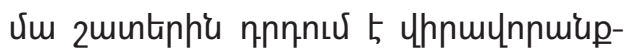
utip huugutal, pnnpnpnus 5 qujpnıрn,

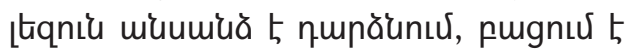

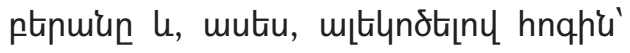

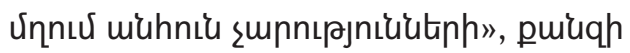

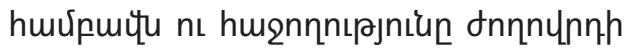

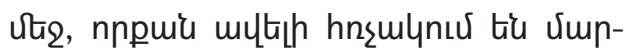

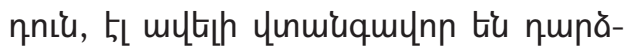

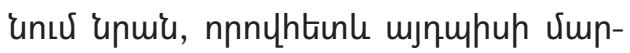

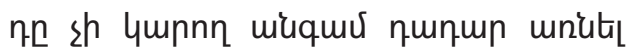
nı mquin 2ustil niatiumal mjnuhup

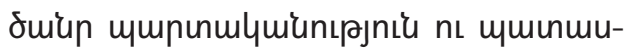

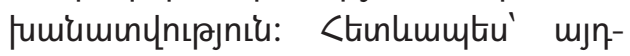
mhuh vimnnn, ptilntqu luinump huqunuunn purp qnnotin, ulio ndumpnt-

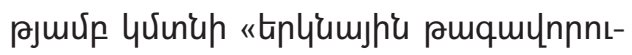

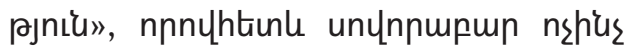

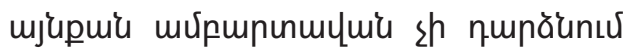

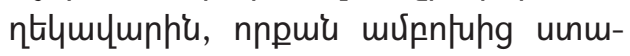

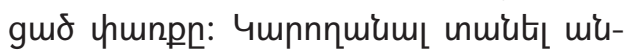

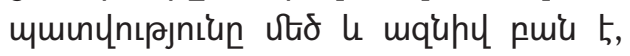

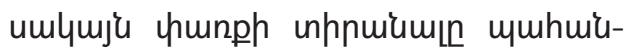
2nıu 5 ynnny u surhmquiug ulio hnqh, nnutiuqh niutíum[ny npu'up' ympnnu-

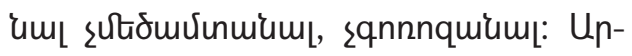

1. ᄂnแ่u untinnuर, t, 134:

2. Unแงu untinnuर, เू 135: 


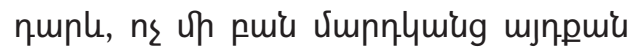

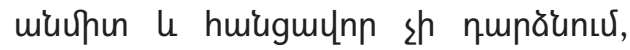

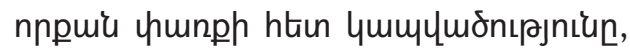
u nshius unuing multih hunquindwiu nt

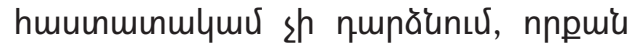

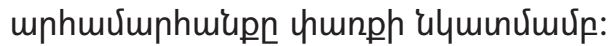

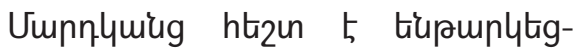

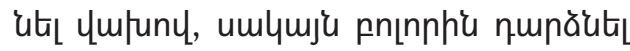

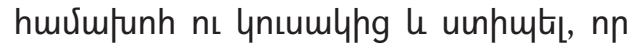
unwiup mulytinonntiu unpurumnnytu h2-

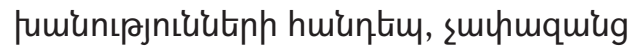

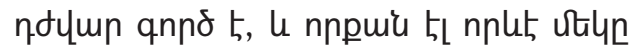

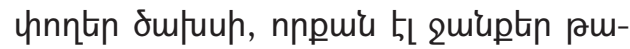

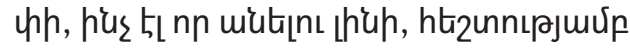

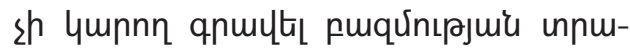

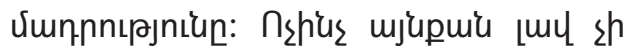

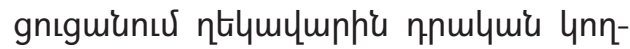
ung, nnpwis 'unu' tiupmlqu'utiph ulymun-

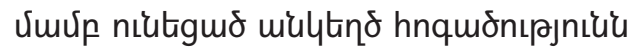
nı hunquiupn:

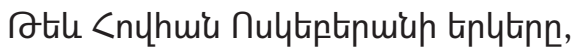
humlqumtu, unm nunnmian Guntinu

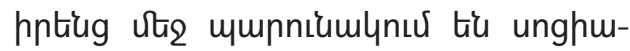

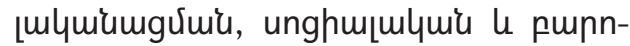

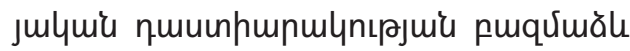

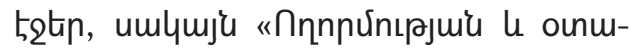

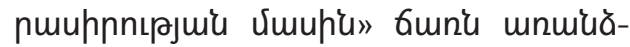
umiunus 5 hiusutiu hn ptưumonhl pnumiu-

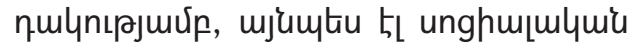

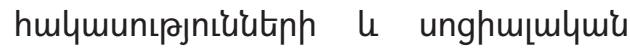

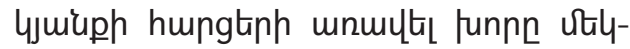

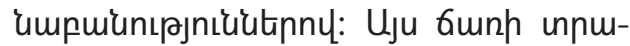

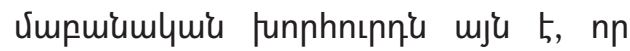

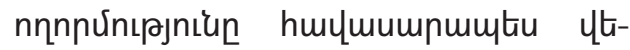

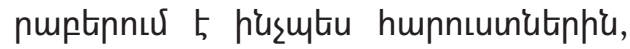

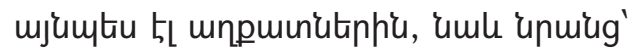
nuptip munts tiu le munnnu tiu nnnn-

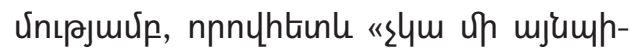

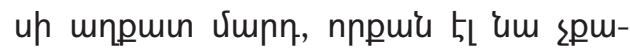

Unn thü, nn sniütium ptilynıq u tiplnı qnn2: Ujn umuunfiunny pshg phs unЦnnn yunnn 5 multah punăn thutal unuinghg,

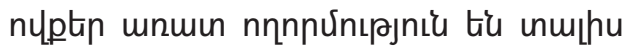
pntiug utio niutigyumophg ...»:

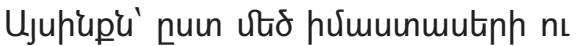

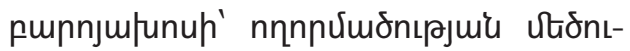

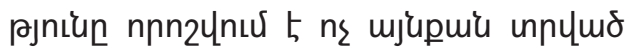

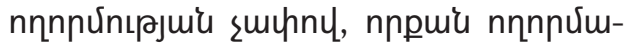

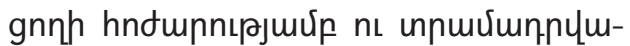

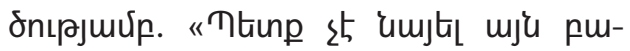
upu, nn mjnhu tiplnn nnưu 5 unmahu,

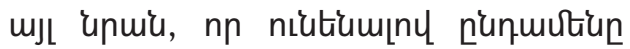

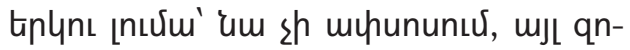

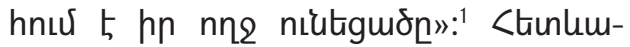

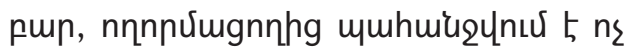

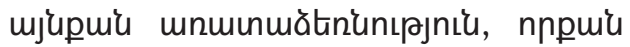

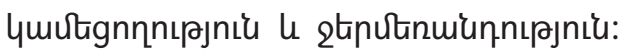
Ctinlunıpjniun, nuin purănuuunh6uiu unn'umunnh, mju t, np tapt yuultgnnnt-

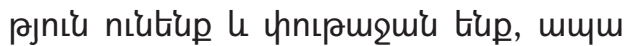
ns un घumu slym mnpmin thitignt ulis, hul tipt sniutiup, mщu hupuinnıpjn-

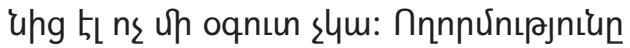

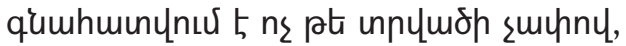

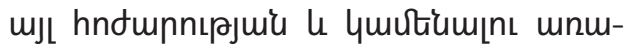

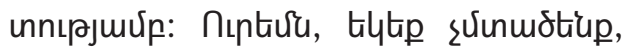

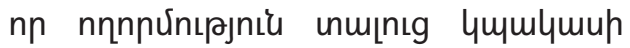

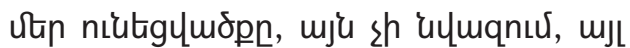

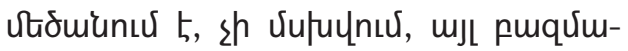

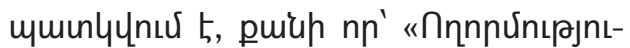
un lumptiu pt hius-nn qnnopp 5 yuu

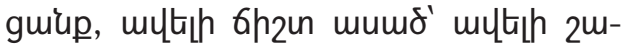
hulltion nt miulunwiug lí mnmghung, l' tinunnnn-hg»:2

hn funup's mqntighl no muunltinu-

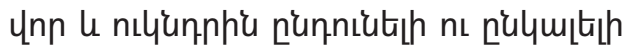

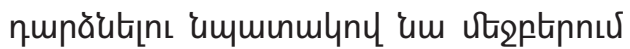

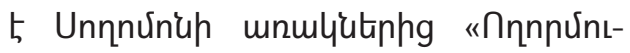

1. Unıju untinnư, to 148:

2. Unı่u untinnuর, t2 151: 


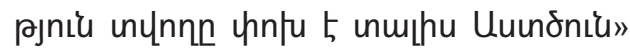
(Unmul. 19, 17) mnwly le npuí unushu 5 junhurhhцhunchujulymí ñunhwiunu-

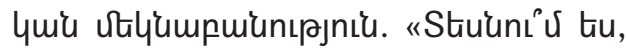

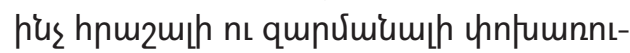

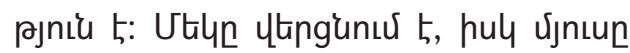

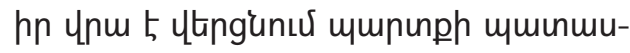
fumiumunЦnıpjnı'un: hǔsnı́ 'um ş munıu'

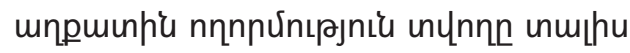

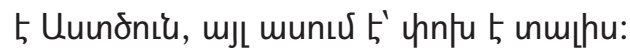

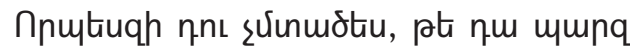
unnfuhwunnıgnuर t: Unınp 9pnpn qhunt

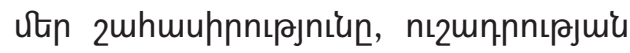

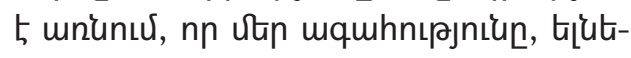

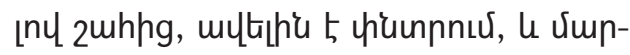

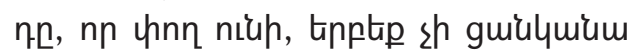

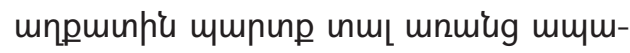
hnunıpjuil, unwing qnuulh, tnmifum-

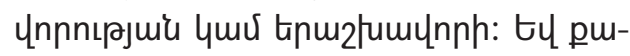

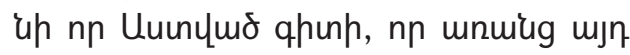

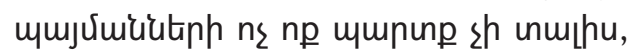

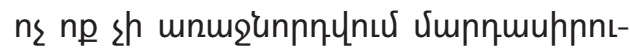

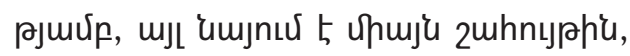
upušntin unpwinn mjn pnцnnhg qnınl

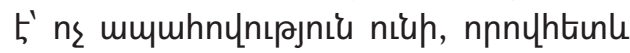
nshius sniuh, ne qnux ympnn 5 nutis, nnnuhtinlu nusumqnınl 5 , ns tnu2fum-

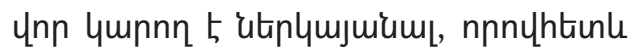
unpwinnıpuid muinfumny unwir ns

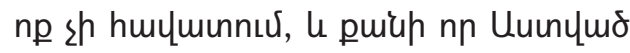
qhunh, nn ytanghiu yunwiugh t5 tiupunlyunıu unpuunnıpjuiu, pul unn niuts-

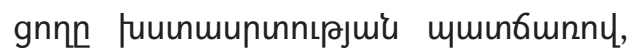

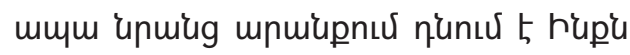
hntí' nnmtu unpuinh hưump tinu2-

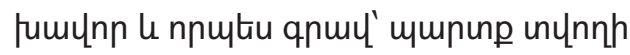
huर्ump»:1

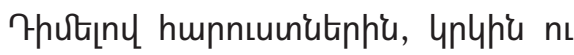
unlhu Gum unuiug hnnnnnnuर $5 n$ nL hu-

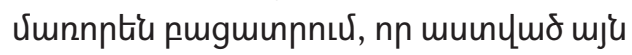

puiuh huvium ss ătiq nıph2utinhg zuin

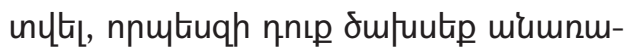

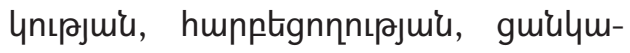

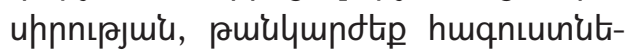

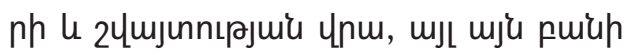
huxump, nn unmp цmphpmцnnitiphis:

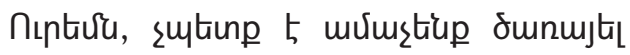
unpuinutiphu, sutiunp 5 hpurdunltiup

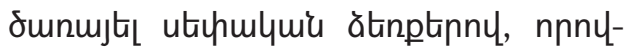

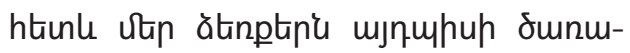

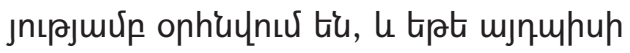

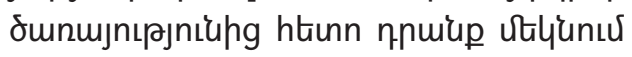

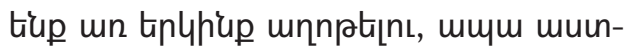

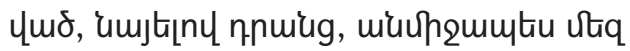

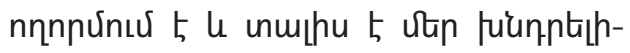

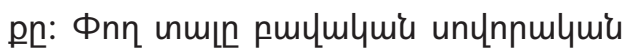

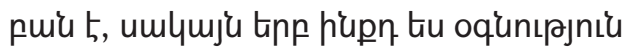

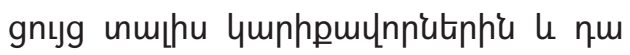

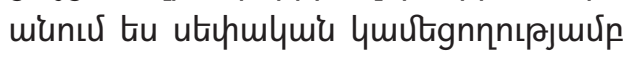

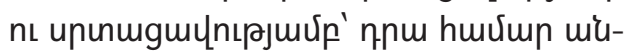

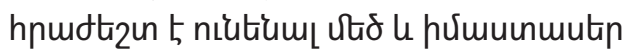
hnqp: ঢjutiny hn unnumumiu, uhphun-

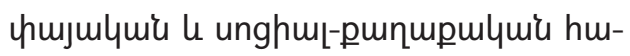

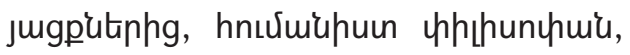

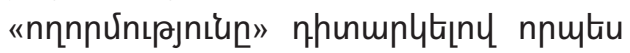
unghu[ulymiumgưu'u qnnohp, ăqunnuर tn hus-nn ălend mju dunnujtightă un-

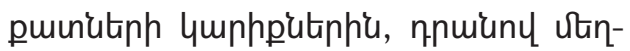

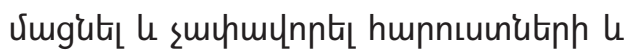

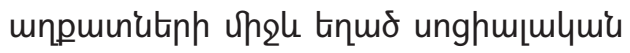

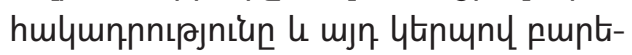

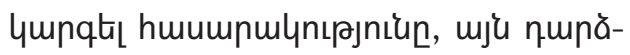
uts phs pt zuin onhumulnn, unnupuigh ne Uumnuutin:

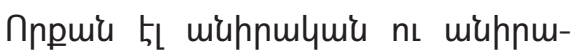
qnnotsh thuthes unu qunumumputs-

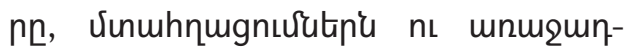

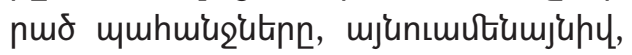

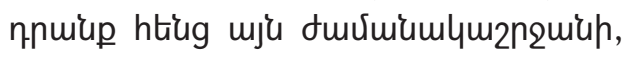
nnh utis munts 5 nultiptinuiun, lu noun-

1. Unเ\}่ untinnư, to 155: 


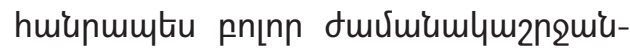
utiph huvium niutigta tiu, niutíu le lnt-

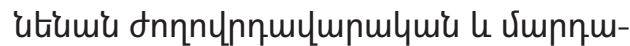

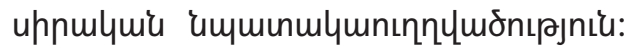

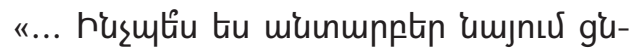

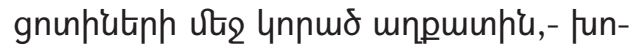

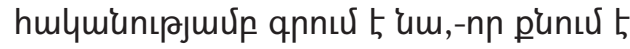

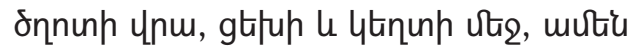

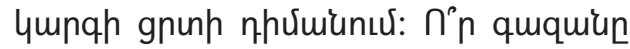

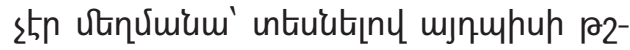

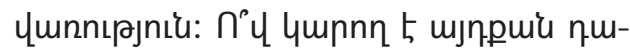

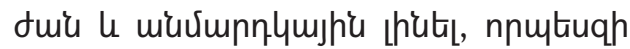
¿nunim funiump no lumbilhg»: Ujupis

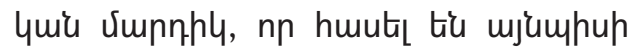

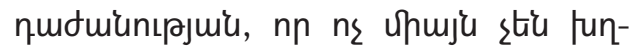
6nux, stî́ gurlulygnux lu ptiplemgunus

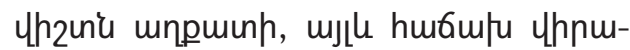

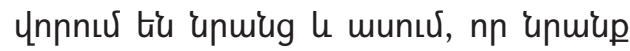
undwiah tín nnuiu: Otapluu mju muinGunny, nn unwiup guidymiunuर tiu ltapulynutal le ne pt unlhg utinutis: "ח's,

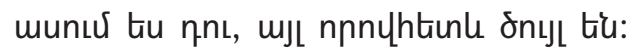

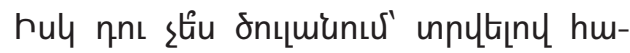
6nuputaphu: Ujumpup qnnotinny stiu qpununuर्u, nnnup guiulquguro unpu-

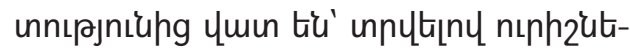

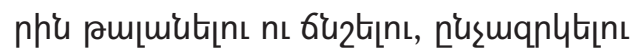

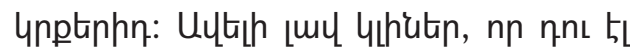

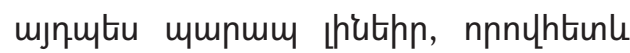

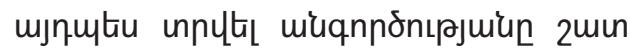

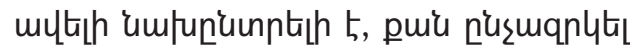

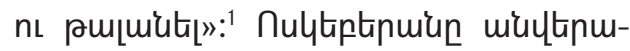

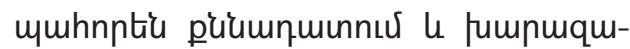

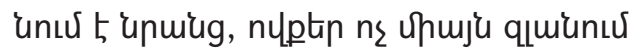

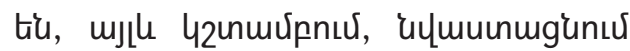

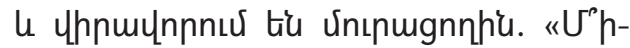

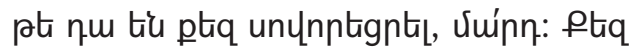

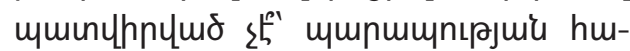

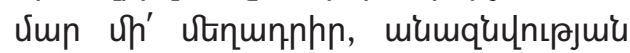

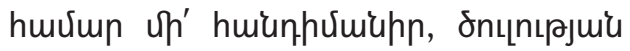

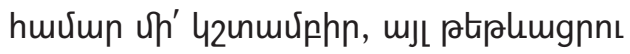

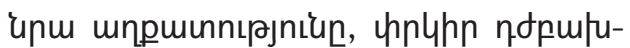

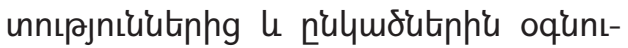
pju'u ătanp uflyuhn, nıptưu un' humgnn unu ljuiuph nı qnnotiph vimupis, tipp

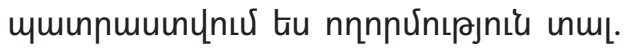

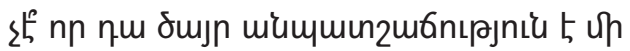

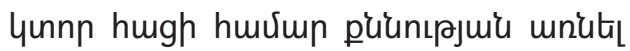

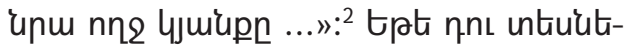

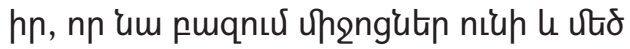

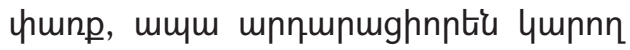
thp mjnutu ympltal, pujg tipt untiuunıर tu, nn üm ndpufuin 5 , up mum, nn

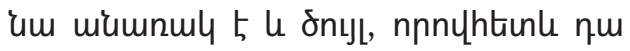

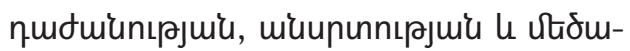

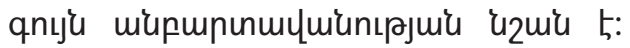

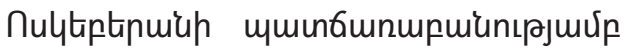

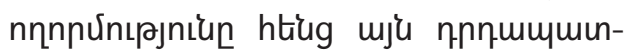

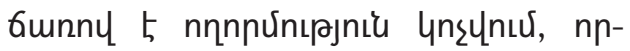
mtuquh uliup nnnnưuiup lí mpduiumunnhu, l' miumpduiututinh's, nnnyhtionle

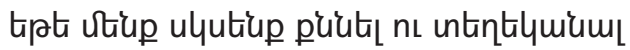

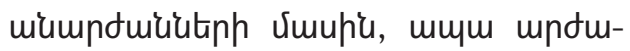

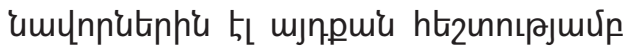
stiup qunuh, hul tots nnnpuluiup umle

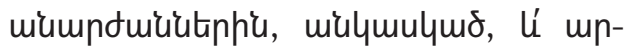
dmiamunnutitph, l' muliu ympq úmpnquing th lhwiunhultup: กy purjugum-

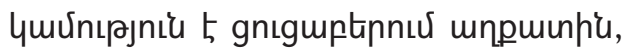

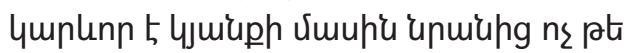

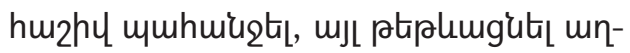

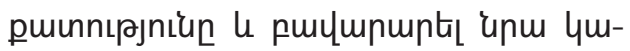

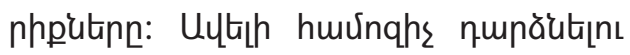
hn Uunptinn 'um' ylymjulynsnuर 5 htint-

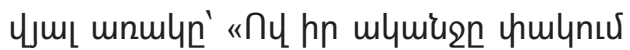

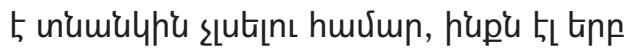
quiush Shnnen, Stinu st hntiu sh Lup» (U-

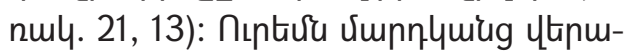

1. ᄂnแju untinnux, t, 169:

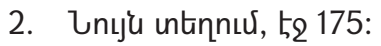




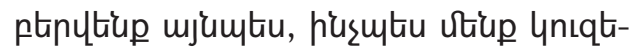
hup, nn Uuunymo ympltap uliq htin: Um

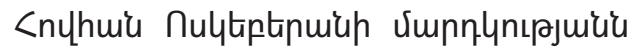

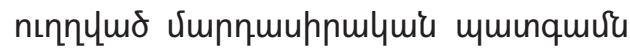
5n nuptiph htinniulatphg:

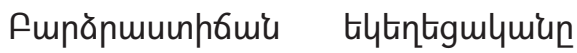

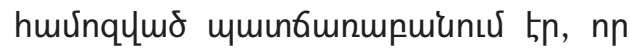

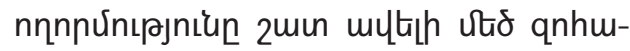

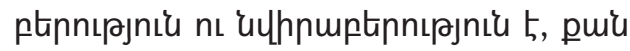
unnрpp, umun lu vjnıu puititnn, tipt,

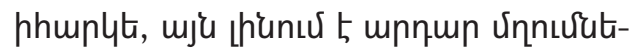

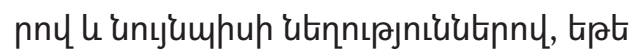

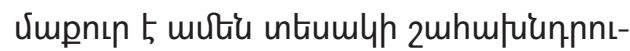

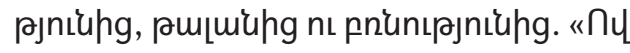

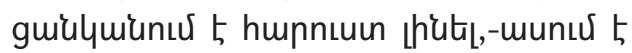
um,-up2un t5 unnnh lumphp 5 qqnuर, pul ny sh guidquiuntu hupniuin qunium,

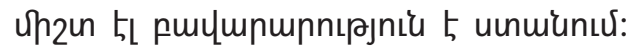

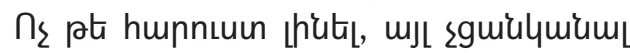
hunuunuiuml, whw pt nnit 5 hupuunntPjniun: Ophiumly huvium mutuर, nn ym

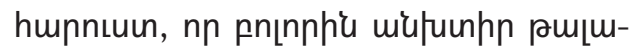
unsu 5 , lu yu hupnsuun, nn hn hupu-

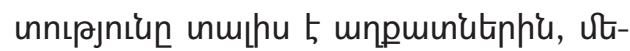

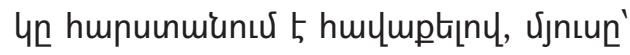

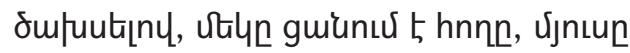

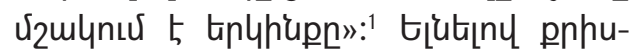

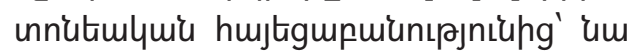
punnqnuर 5 vimpnlyming htinns vaumL pulwuhg, mquhnıpjniuhg, qnump-

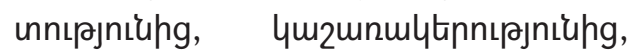
mupupn upp nt pupphg lu pugmum-

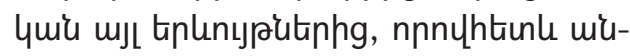

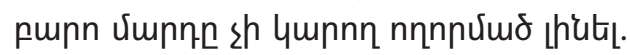
"tpt utiup upliunıju ătinptinny l nıph2-

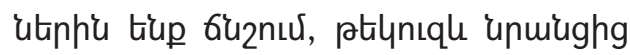
ytrngnuorny huqgutiup nıph2utphi, upliuny'u 5 , utiup muinohg stiup funs-

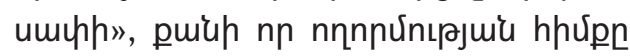

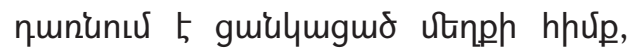

1. Unıju untnnıu, t2 177-178:

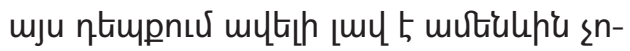

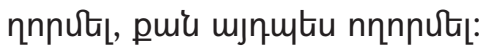

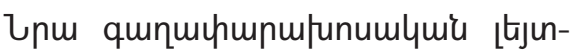
ununhyl unghmimlymir huцluumpnt-

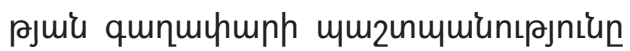

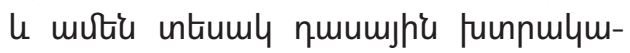

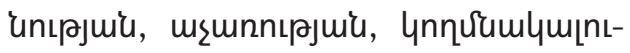

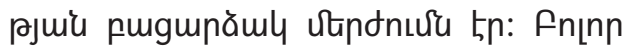

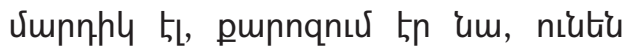

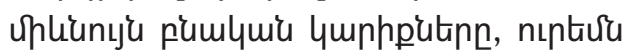
u npuing purlummentins ungngutinn muliu untin hurlumump mitung 5 Lhitiu:

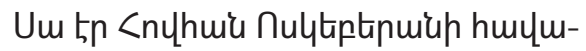
unn huirquiumlyn:

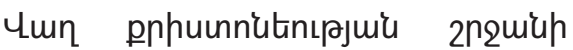

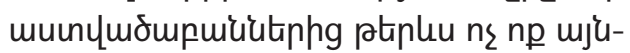
puiu funne sparhwingtig unghm[mumir ujuiuph hungtiph ulig $4 \mathrm{~h}$ yh6ulyh sta-

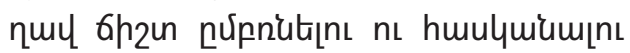

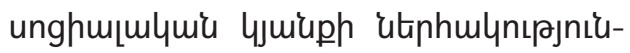

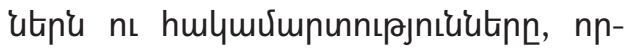
puiu <nųhuiu nulthptinuiun: lu huiu-

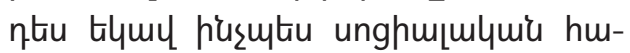

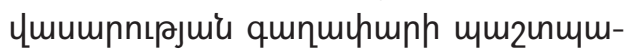

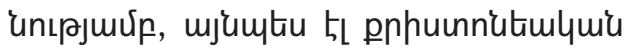

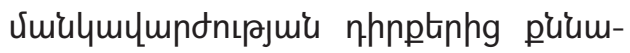

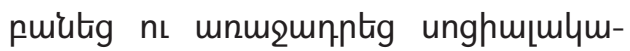

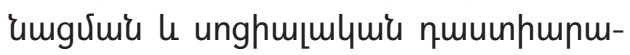
ynıpjui hungtin:

Unu nuinnuiuh Guntiph utis hp

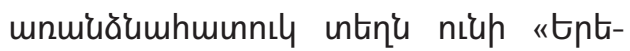

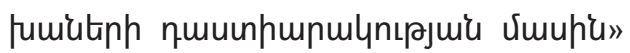
Gunn, nnuntan ưu punănugưunư 5 tipti-

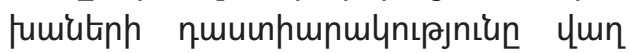
unuphphg ulyut_ns umbhwiugn: Fn[nn

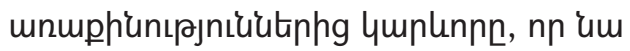
muhwiugnus tn quaunpurmults tipt-

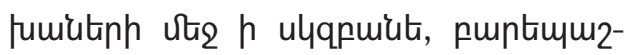

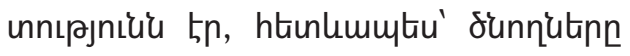
ne pt utiunp 5 vinmotiu mju Umuh'u, ph 


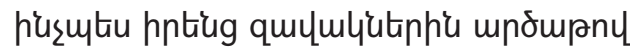

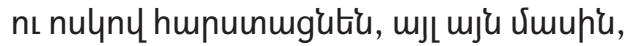
pts piscutu unuiup nuniumiu pn!nnhg

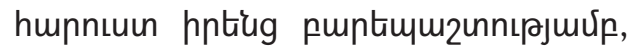

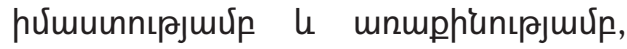
nıntiư, mitunp 5 quiumnnupup htinlits

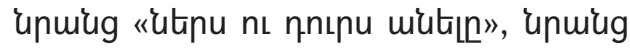
"qnnıgühtiu nı huцuputinn" u pugu-

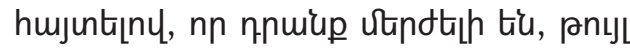

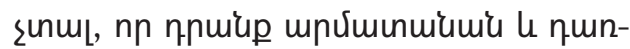

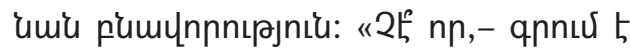
uim,- tipt ufiquhg huzhy 5 muhwiug-

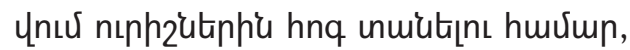

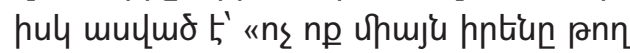
¿4huunnh, mJ umule nulutinnghun" (U. 4nnip. 10, 24), шщщu nnpwis multah 5

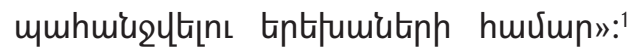
Uhisntin muliú hius miunsu tiup, nnutiu-

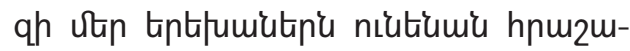

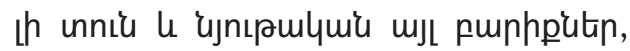
umlumju mutiulupis muntiunus tiup $u$

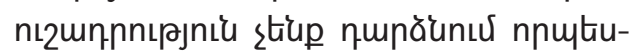
qh unmiap niutiumis juy hnqh lu punp luuvp: Um pur huurnqumo tn, nn ptynıqu ulilh niutigumon utio nt puilympthp thuh, pujg tipt um muinnmuin st

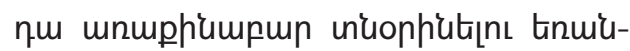

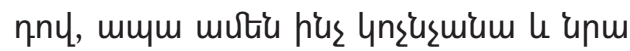
htin yltinuium, hul tipe hnqha mqualu-

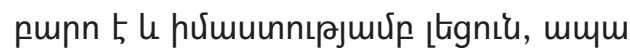

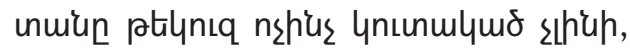

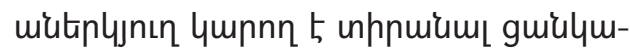

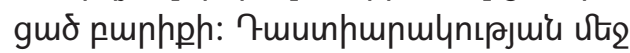
nultiptinuiun stn utindnux muinhoduti-

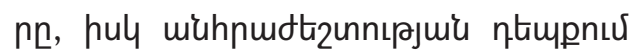

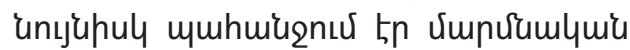

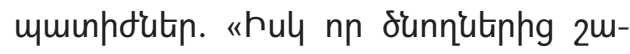
unten,--qnnuर 5 'um,-2min puís tiu pu-

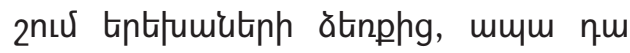

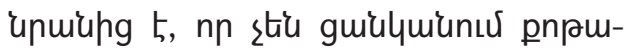

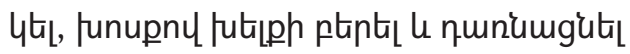

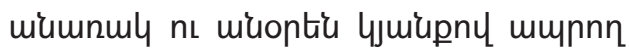
hntiug quilulyitiphis, mjn muinfiunny phes sh muinumnux, np unuiup mlquium-

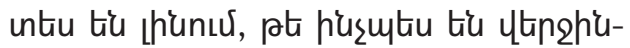

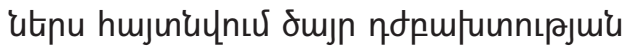

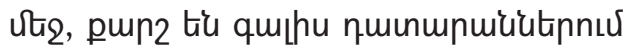

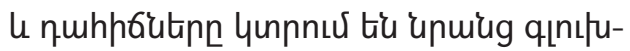
utinn": ${ }^{2}$

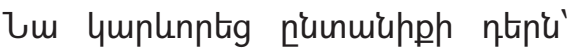
hpple unghmemlymiamguime humunmunnıpjnı'u, L qunqugntig mju upunpn,

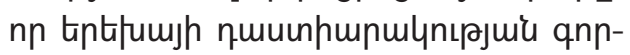

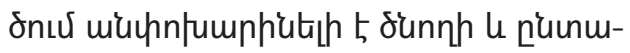

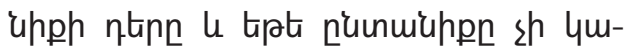

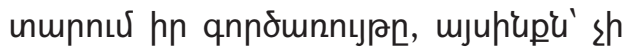

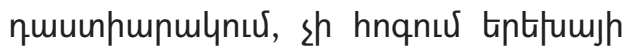

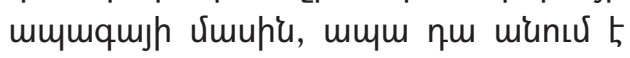
tiptjumjp huvium minpuptiummuin un-

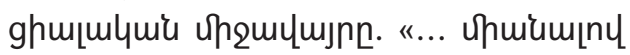
unplumitiphis nt unsuguro vimpnlyming

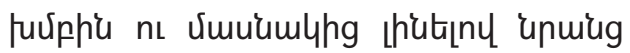

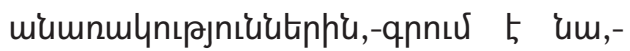

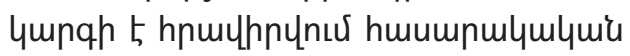

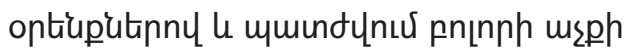
unme, huly verhmumundhe htinlunus 5

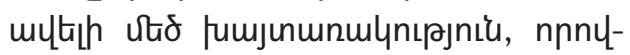

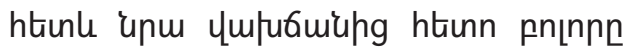
Uumunumgnug tiu miunıu hnnL":

Multiptipuiun mưnnununătă

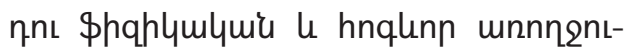

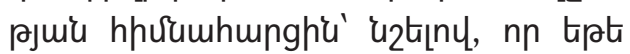

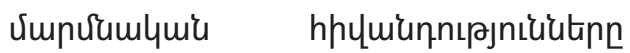

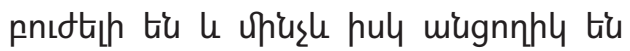

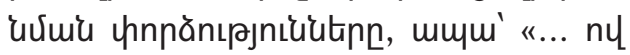

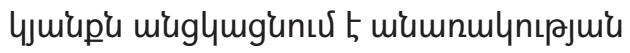

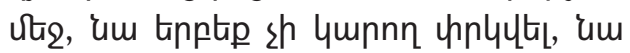

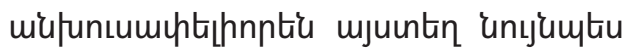

1. ᄂnเ\}u untinnư, t, 185:

2. Unแยน untinnเư: 
mpduiuh 5 miampquimiu, hul mjuintinh

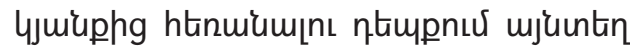
uny'umbitu qundwiamiam hulhuntíum-

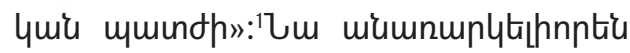
pưumnuuntig dunnulymiu miunt2unnnt-

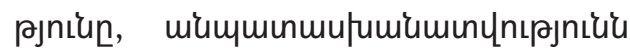

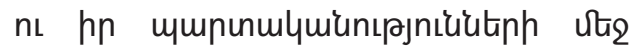

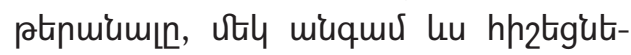

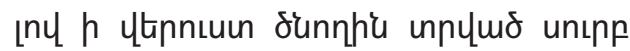

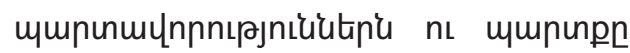

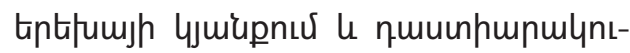

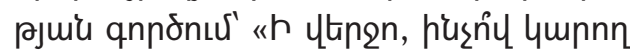

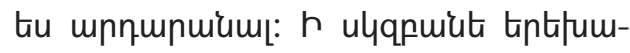
utphis ptiq htion muntal sltapumuhtgif ntin ymulh ptiq: Unuiug quluhis ptrq niunıghs ymnqtigh, nuuunhunuly,

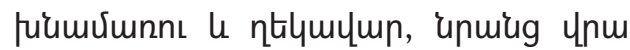

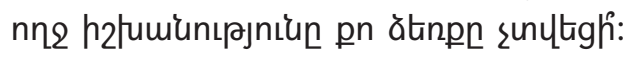
Ujnpwiu p'upnเ2 tulyh'u slympqunntigh

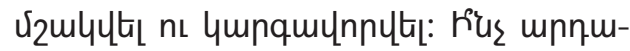

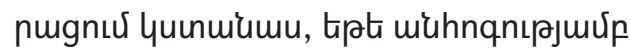
umjtu unm pnslynunnưutphiu: rius ym-

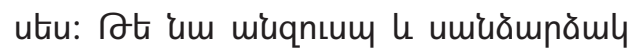
5n:2 fujg hunl 5n, nn mృ mutiuhu htiug ulqqphg htiunltipn' um'uătipn, tip um ntin unnp tn nı umiăh tiupalym,

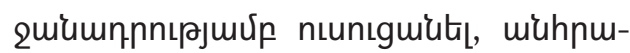

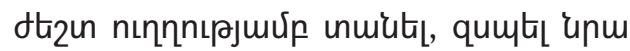
hnqtiquiu unnnplynưutinn, tinn um ntin

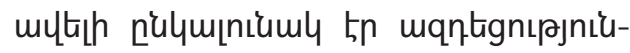

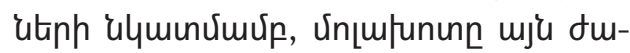

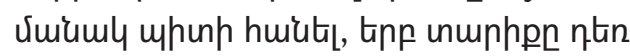

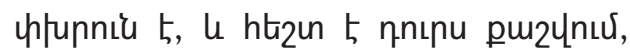

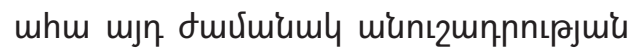

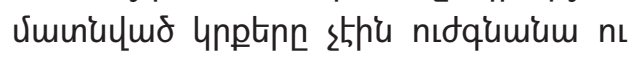

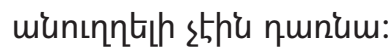

Um fumpuquiunsu th sunnulquiu

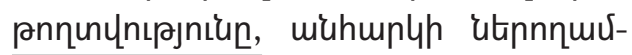

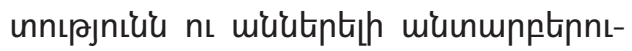

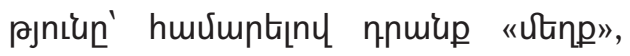

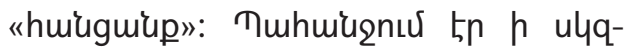
puits tiptipumsh utis quuunhmpulyts

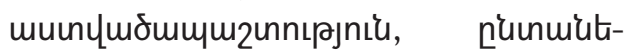

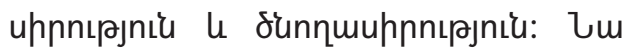

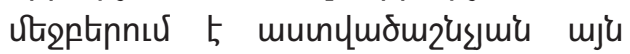

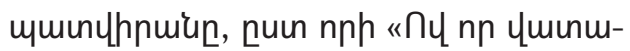

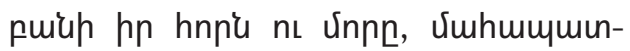
op pnn tüumplylh (七!p, 21, 17)», hul

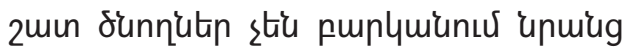

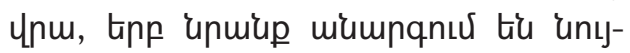

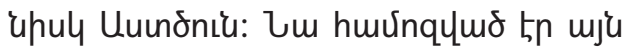

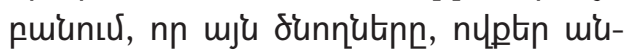
unsus tiu hntiug tiptifumitiph huiuntiu, ptlynugle ujnıu hungtinnux unmiup juy

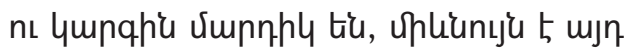
"ulinph», mjn "huiugmiuph" huvium undu'umium[ns tiu miumpquiuph' "huly tpts nıqnuर tp 6hzu'u hưuium, np 'unıupuly mju duviumumly, tipp utip vjnıu

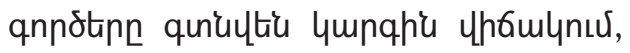

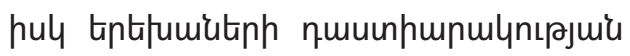

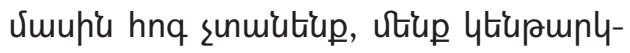

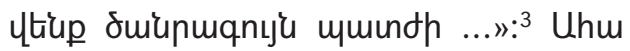

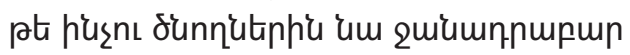

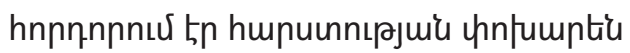

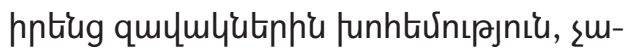

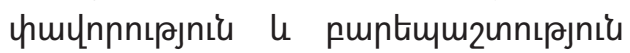
dunuiuqtiu: lu dmjnuhtin miuvinnt-

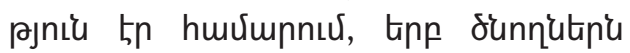

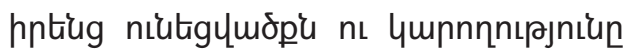
dunuiuqnuर the ne pt ytiunuiunıpuiu durumiuml, mJ hnting vimhng htionn' пnuiuny uto

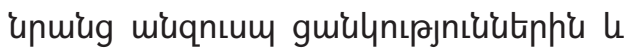

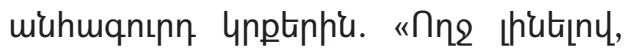

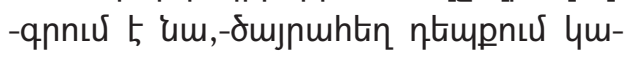

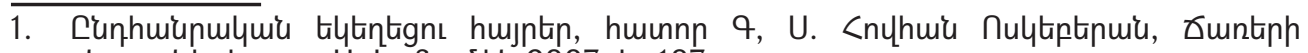

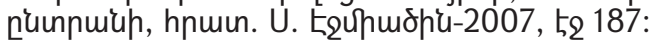

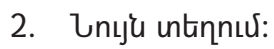

3. Unเ\}u untinnux, t.2 188: 
nnn thip unuinghg huzhy muhuiugtal,

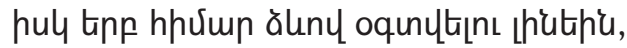

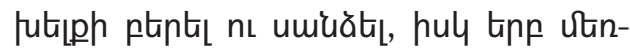
utiup, mum ufiquiung qnltinny l unming uniophunısjuí unuly annitinny tiph-

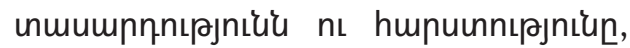
unwiug unnux tiup wühw2hy untiunut-

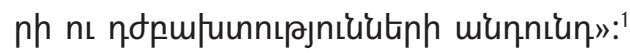
nıptưu, utiunp 5 ăquntiup ne pti hupu-

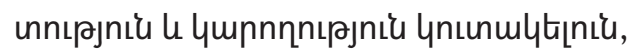

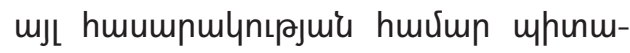
up la mondmiammuinhy punupmghitin

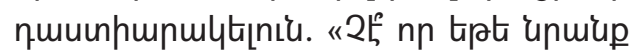

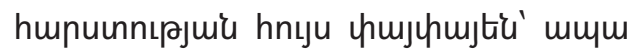

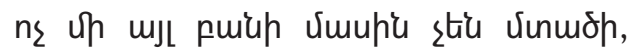
nnnuhtiunl unnnh thnıpjniun unuiug msphí liuutúuguh hntiug punptiph mpuunuulnn shutisn, huly tipt untruitiu, nn

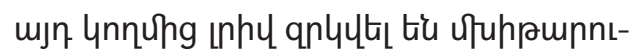

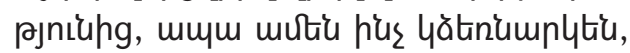

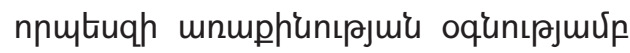

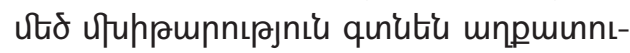

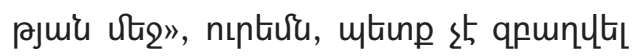

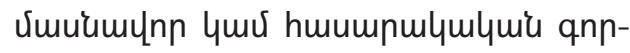

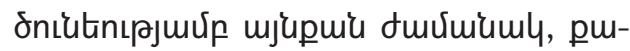
up nten ulip quulmlyutiph vimpnlumjhe

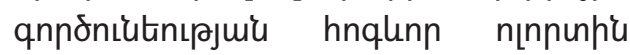

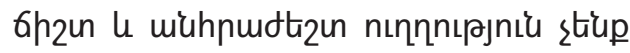
unlta, nnnyhtiunl' «tpts nnıp quuunh-

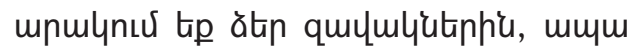
unwiup hnting htipph's quuunpunuynuर tiu hnting quululyitinhu, hul mju

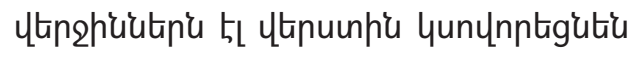
pntiughiuatinha' mjnutu zunniuml..., u mjn qnnop nng munqun yhuuuh unwiu,

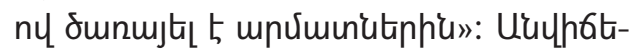
th 5, nn <nyhuir nultiptenuiun pugumhujun fununtú 5 unghmimlumu thnnăp'

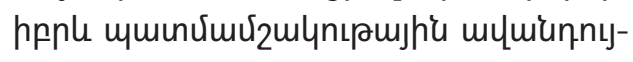

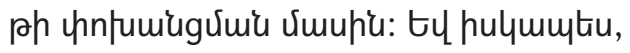
tipt juy tu quuunhwnulyt pn nnnniu, hul 'uw' hn nnnniu, huly uw' pntiuh'u,

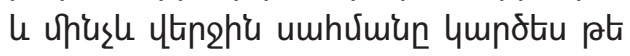
ăqunuর 5 hus

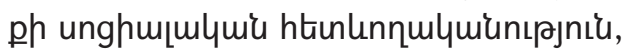

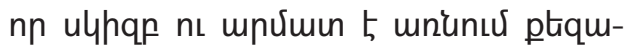

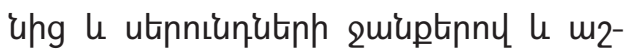
fumunuiupny ptiq unujumtiu huuguntu 5 hn munnınutinn: Unu htinlenıpjnıนun

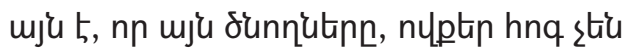
unuiunus phting quilulyitiph nuuunhu-

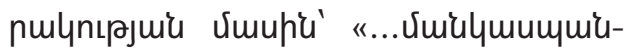

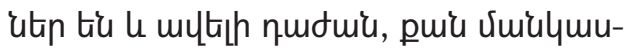
muiuktinn, pwiah nn mjuintin funupn

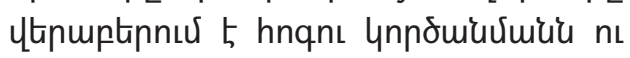
Uuhulu'un»:2

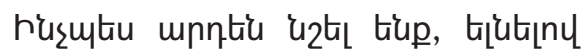
hn unn'umlymín nnquiuiutingh, nultipti-

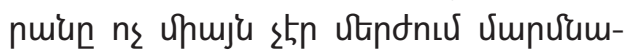

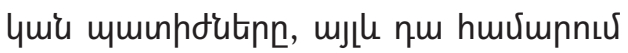

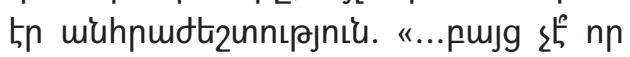

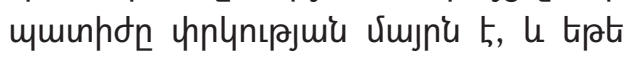

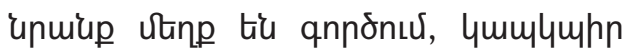

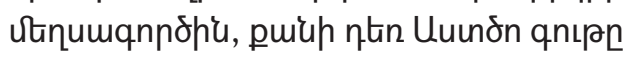

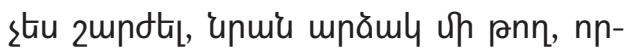

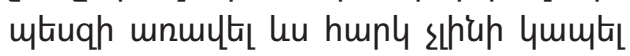

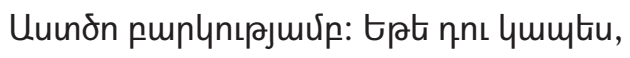
Uuinumo mjunchtionle sh qump, hul

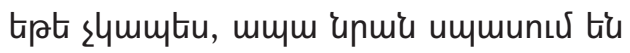

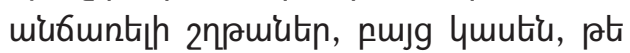

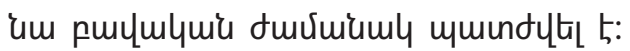
<ungunuru tư' híspuîu: Utily unuph, tip-

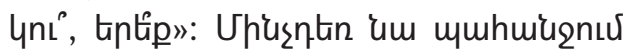
tn ns pt durumumuly unlumlymunıpjnıน,

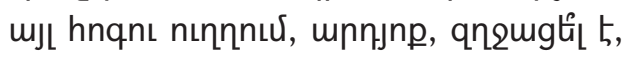

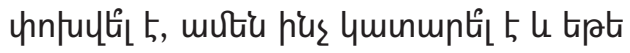
mjnutu ss, mum ns up oqnin slym duvimiumlyhg, puiuh np' "...utiup muhwiu-

1. ᄂnแรu untinnuर, t, 190:

2. Unưu untinnuu, t, 191: 


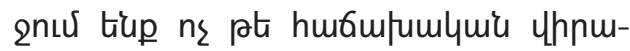

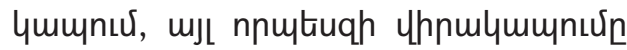
hüs-np oqnen unu, tipt mju oqunulyum

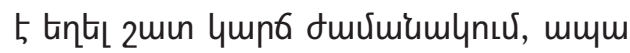

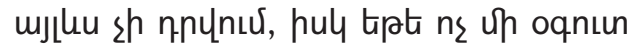

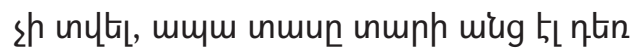
mtinp 5 n.

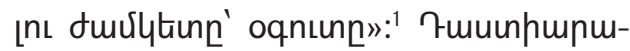

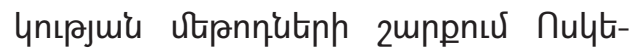

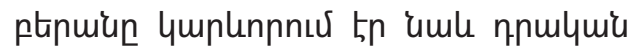

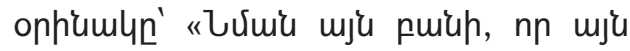

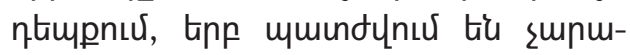

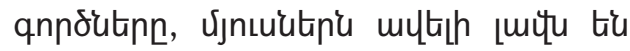

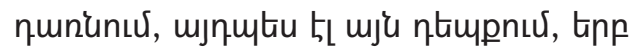

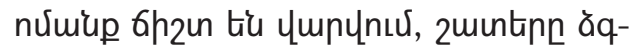
unnuर tiu nunonhumbti| unuiug»: Fujg $\mathrm{u}$ mjumta, nulktptinuiun qtpmumunynt-

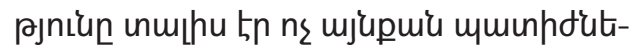

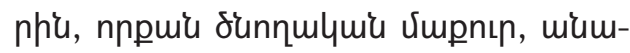
umuluiu uhnniu, nnnyhtiunli "...nshius

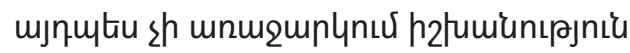
niutgnnn, puiu qtinumqhí utn un tiu-

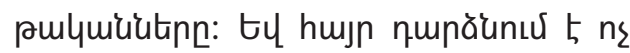

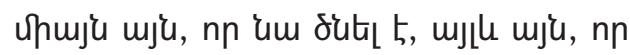

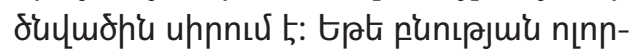

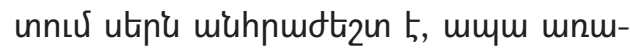

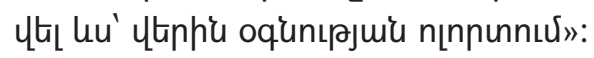

funnănumunnfruí hnqunnulquiun

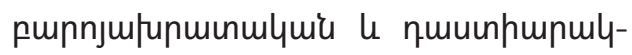

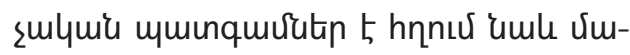

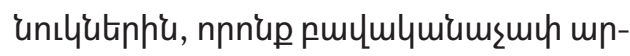

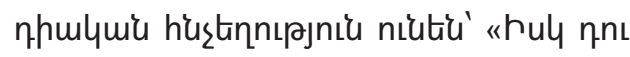
Juiulyhl, humquiunpn ptiq dunnhí...: hǔsñy uhunh humnnıgtu mju, hus ptiq

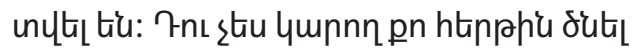

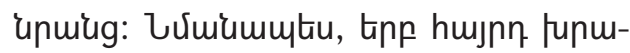
unnus 5 tinpnnn, upm htion qujnughn

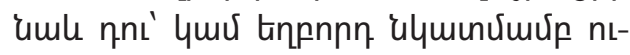
'utguro hnquinumnıpjniuhg, 4mư hnn

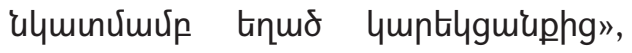
nnnuhtiunle tipt utinuulnne untuuh, nn hujpu hntiu umuindnuर 5 , hul ynnphg

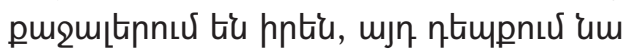

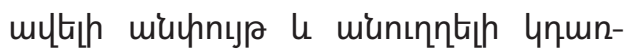
um, hul pmomitinnn'u hn ynu 4ltingup humnntgnuún, puiuh nn' "...'um, ny

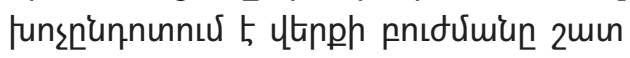
multh muindh 5 mpdwiuh, puiu 'um, ny huugnts 5 ytanpn, npnyhtiule unyju puun st' yhnuunnts l fumiqumpts ytanph pnıdumiun, ytanghun ytang'umlymiummtu huiuqtigunus 5 vimhluiu, hul mnmgh-

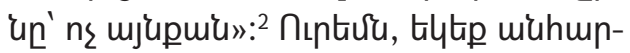
quing suhutiup dunnitiph ulumunumurp, nnnuhtinle tipt unuiug hunquiupny

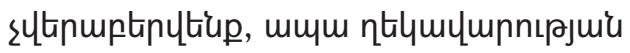

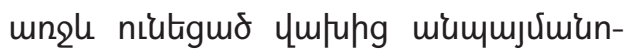

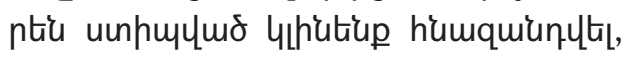
hul tipt utinp qnnotiny uphuxumphtiup unwing, шщш tipptp stiup ymnnn funcumicts funth fumjphg, tipt npuiup

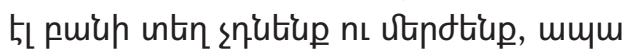

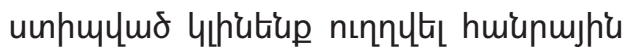

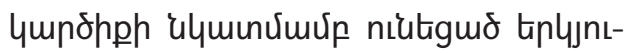
nhg, tipts um th henhumunipjniu st,

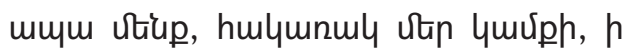
Uh6ulyh tiup shitinı unwiu futeph ptipts

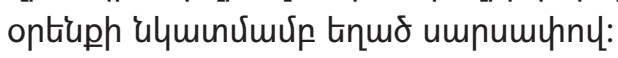

<nyhuiu nultaptamiun miannumun-

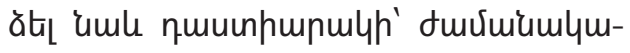

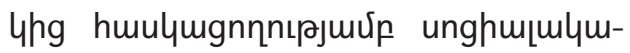

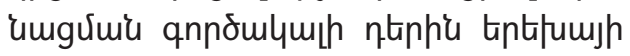

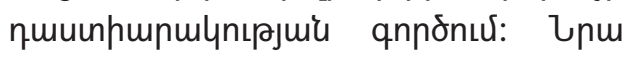

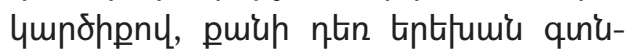

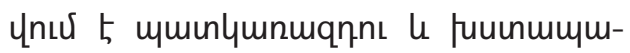
huias quuunhumulyh ätnpp unuly, qunviuiup sh hupnıgnıu, ptilnıqu funumph thup, ptilnıqu munltizon ujuí-

1. Unıju untnnuर, t5 191-192:

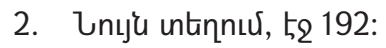


pny munp, umlymju muinmians funäum-

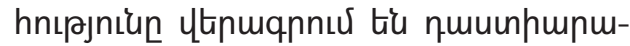
uh ulquinúurup niutigmo hmpquiuphu ymu ymupu, hul tipn quuunhmpulyh

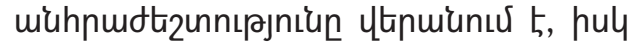

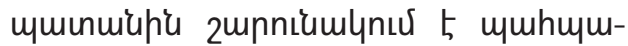

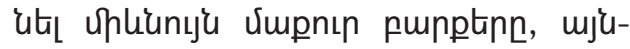

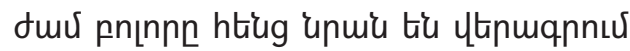
uml Gumpunn

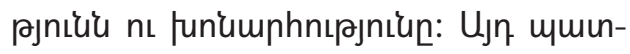
Gunny, nuin unm, huply 5 nuuunhu-

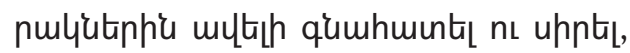
puiu dunn'utiph'u, puiuh np' «...uju पltp-

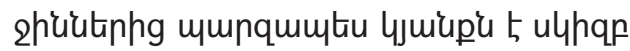

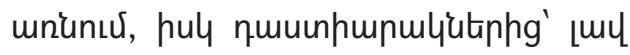

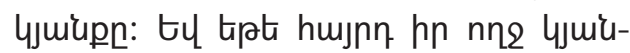
pnux unıpp 5 tints, uf upplhn, htiug nu ptiq huviump quinumumpunumiu unhp yhuiunhumiam, tip unuiu'u niatiumany

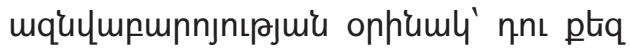
umpumhujptiphn unwphiunıرu'u huiu-

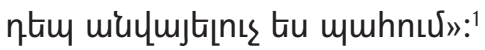

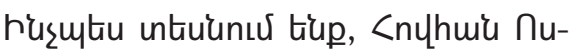

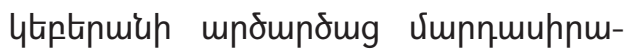

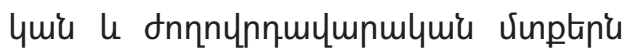

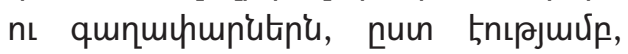

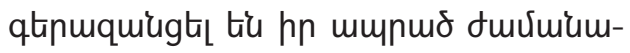
ymanquiah zmin intumbtinutin, ymp-

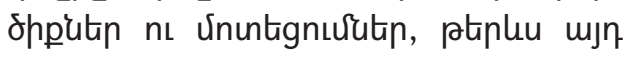
muinfiunny th nnuiup nnıpu tiu tilts durumiumb tiqnuqdong $u$ hntiug npu-

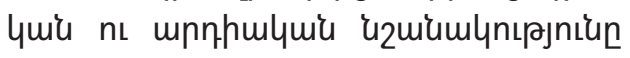

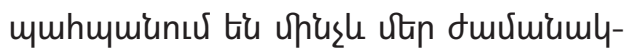

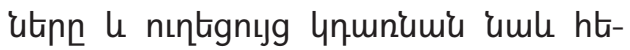
unmqu duviuiumlititph huviup:

\section{ВОПРОСЫ СОЦИАЛЬНОГО ВОСПИТАНИЯ В СБОРНИКЕ СОЧИНЕНИЙ «ИЗБРАННЫЕ РЕЧИ» ОВАНА ВОСКЕБЕРАНА}

ШОГИК ВОСКАНЯН

Профессор кафедры дошкольной педагогики и методики Армянского государственного педагогического университета имени Хачатура Абовяна,

доктор педагогических наук

Античная фрилософрия, в частности Платон, имели слабое влияние на произведения и мировоззрение богословов ранней христианской эры. Платон сумел соединить идею и материю в одном, общем мире, который был одновременно и чувственным, и материальным, и идеально гармоничным и красивым. "Отцы церкви» использовали эллинистическую философию лишь в качестве инструмента и метода для того, чтобы ознакомить и на доступном языке довести до своих современников основные положения Христианства. Одним из самых выдающихся «отцов церкви» был богослов, писатель и ритор Ован Воскеберан, который выступал за защиту идеи социального равенства и анализировал вопросы социализации и социального воспитания с точки зрения христианской педагогики.

1. Unเงน untinnữ, t, 193: 


\title{
ISSUES OF SOCIAL EDUCATION IN THE COLLECTED WORKS «SELECTED SPEECHES» BY HOVHAN VOSKEBERAN
}

\author{
SHOGHIK VOSKANYAN \\ Professor at Kh.Abovian Armenian State \\ Pedagogical University, Chair of Pre-school \\ pedagogy and methodology, \\ Doctor of Pedagogical Sciences
}

\begin{abstract}
Ancient philosophy and Plato in the people of their era in a easy to particular has had a weak influence on the understand and simple manner. One of works and worldview of the theologists the most prominent and significant of of the early Christianity era. Plato had these "founding fathers of the church" managed to unite the idea and material in one ecumenic world which was both sensual, material and ideally harmonious and beautiful. "The founding fathers of the church" used Hellenistic philosophy only as a tool and a method to introduce was theologist, writer and rhetorician Hovhan Voskeberan who supported the idea of social equality. He also analysed and highlighted the issues of socialization and social education from the standpoint of Christian pedagogy.
\end{abstract} the main provisions of Christianity to 